\title{
Compound climate risks threaten aquatic food system benefits
}

\author{
Michelle Tigchelaar ${ }^{1,20 凶}$, William W. L. Cheung ${ }^{2,20}$, Essam Yassin Mohammed ${ }^{3,20}$, \\ Michael J. Phillips ${ }^{3,20}$, Hanna J. Payne ${ }^{\mathbb{1} 1}$, Elizabeth R. Selig ${ }^{(1)}$, Colette C. C. Wabnitz ${ }^{1,2}$, \\ Muhammed A. Oyinlola $\mathbb{D}^{2}$, Thomas L. Frölicher ${ }^{4,5}$, Jessica A. Gephart ${ }^{6}{ }^{6}$, Christopher D. Golden ${ }^{7}$, \\ Edward H. Allison (103 ${ }^{3}$, Abigail Bennett ${ }^{8}$, Ling Cao9 , Jessica Fanzo ${ }^{10,11}$, Benjamin S. Halpern (10) 12,13, \\ Vicky W. Y. Lam ${ }^{(12}$, Fiorenza Micheli ${ }^{1,14}$, Rosamond L. Naylor ${ }^{15}{ }^{15}$ U. Rashid Sumaila ${ }^{2,16}$, \\ Alessandro Tagliabue ${ }^{17}$ and Max Troell ${ }^{18}{ }^{18,19}$
}

\begin{abstract}
Aquatic foods from marine and freshwater systems are critical to the nutrition, health, livelihoods, economies and cultures of billions of people worldwide, but climate-related hazards may compromise their ability to provide these benefits. Here, we estimate national-level aquatic food system climate risk using an integrative food systems approach that connects climate hazards impacting marine and freshwater capture fisheries and aquaculture to their contributions to sustainable food system outcomes. We show that without mitigation, climate hazards pose high risks to nutritional, social, economic and environmental outcomes worldwide-especially for wild-capture fisheries in Africa, South and Southeast Asia, and Small Island Developing States. For countries projected to experience compound climate risks, reducing societal vulnerabilities can lower climate risk by margins similar to meeting Paris Agreement mitigation targets. System-level interventions addressing dimensions such as governance, gender equity and poverty are needed to enhance aquatic and terrestrial food system resilience and provide investments with large co-benefits towards meeting the Sustainable Development Goals.
\end{abstract}

limate change threatens all aspects of aquatic food systems, from production to consumption ${ }^{1-4}$, endangering the cultures, livelihoods, economies, health and nutrition of billions of people around the world ${ }^{5-9}$. To date, studies of climate change impacts on aquatic foods (that is, fish, invertebrates and algae captured or cultured in freshwater and marine ecosystems for food or feed) have failed to provide a full accounting of this risk, as they have largely focused on individual production systems (for example, marine fisherie ${ }^{10}$ ) and have rarely connected production system impacts to the differential contributions of aquatic foods to food system outcomes ${ }^{11}$. Simultaneously, aquatic foods may have substantial but diverse roles to play in transformations towards sustainable and equitable food systems and healthy diets to address multiple forms of malnutrition, especially for coastal communities and the world's undernourished ${ }^{12-15}$. Efforts to build climate-resilient food systems must ensure that key health, equity and sustainability goals ${ }^{16}$ are met at national, regional and global scales.

Environmental change caused by rising atmospheric greenhouse gas concentrations creates differential climate hazards for the production capacity of marine and freshwater systems, aquafeed resources and supply, and post-production processes (Supplementary Table 11). For example, pelagic fisheries may increasingly have to contend with shifts in species distributions ${ }^{1,2,10}$; coral reef fisheries and bivalve production with ocean acidification $^{1,2,17}$; inland fisheries with the timing and volume of freshwater availability ${ }^{1,18}$; and fed aquaculture with terrestrial crop losses for feed $^{17,19}$. Here, we develop and apply an integrative food system approach $^{11,20}$ (Supplementary Methods) to put all aquatic foods on the same table ${ }^{21}$ and quantify the risk that climate change poses to the potential for aquatic foods to contribute to sustainable development (key terms, including 'risk', are defined in the Supplementary Information)

From literature-identified climate impact pathways (Supplementary Table 11), we selected representative and well-understood variables projected by three Earth system models (ESMs) that participated in the Coupled Model Intercomparison Project Phase 6 (CMIP6) to calculate cumulative national-level climate hazard scores in the near future (2021-2040) as well as the middle (2041-2060) and end (2081-2100) of the twenty-first century under two contrasting emission scenarios. We then integrated these hazard scores with

\footnotetext{
${ }^{1}$ Center for Ocean Solutions, Stanford University, Stanford, CA, USA. ${ }^{2}$ Institute for the Oceans and Fisheries, University of British Columbia, Vancouver, British Columbia, Canada. ${ }^{3}$ WorldFish, Bayan Lepas, Malaysia. ${ }^{4}$ Climate and Environmental Physics, University of Bern, Bern, Switzerland. ${ }^{5}$ Oeschger Centre for Climate Change Research, University of Bern, Bern, Switzerland. ${ }^{6}$ Department of Environmental Science, American University, Washington DC, USA. ${ }^{7}$ Department of Nutrition, Harvard T.H. Chan School of Public Health, Harvard University, Boston, MA, USA. ${ }^{8}$ Department of Fisheries and Wildlife, Michigan State University, East Lansing, MI, USA. ${ }^{9}$ School of Oceanography, Shanghai Jiao Tong University, Shanghai, China. ${ }^{10}$ Berman Institute of Bioethics, Johns Hopkins University, Baltimore, MD, USA. "1Nitze School of Advanced International Studies, Johns Hopkins University, Washington DC, USA. ${ }^{12}$ National Center for Ecological Analysis and Synthesis, University of California, Santa Barbara, CA, USA. ${ }^{13}$ Bren School of Environmental Science and Management, University of California, Santa Barbara, CA, USA. ${ }^{14}$ Hopkins Marine Station, Stanford University, Pacific Grove, CA, USA. ${ }^{15}$ Department of Earth System Science, Stanford University, Stanford, CA, USA. ${ }^{16}$ School of Public Policy and Global Affairs, University of British Columbia, Vancouver, British Columbia, Canada. ${ }^{17}$ School of Environmental Sciences, University of Liverpool, Liverpool, UK. ${ }^{18}$ Beijer Institute of Ecological Economics, The Royal Swedish Academy of Sciences, Stockholm, Sweden. ${ }^{19}$ Stockholm Resilience Centre, Stockholm University, Stockholm, Sweden. ${ }^{20}$ These authors contributed

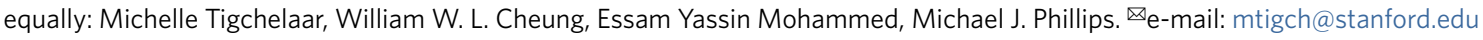



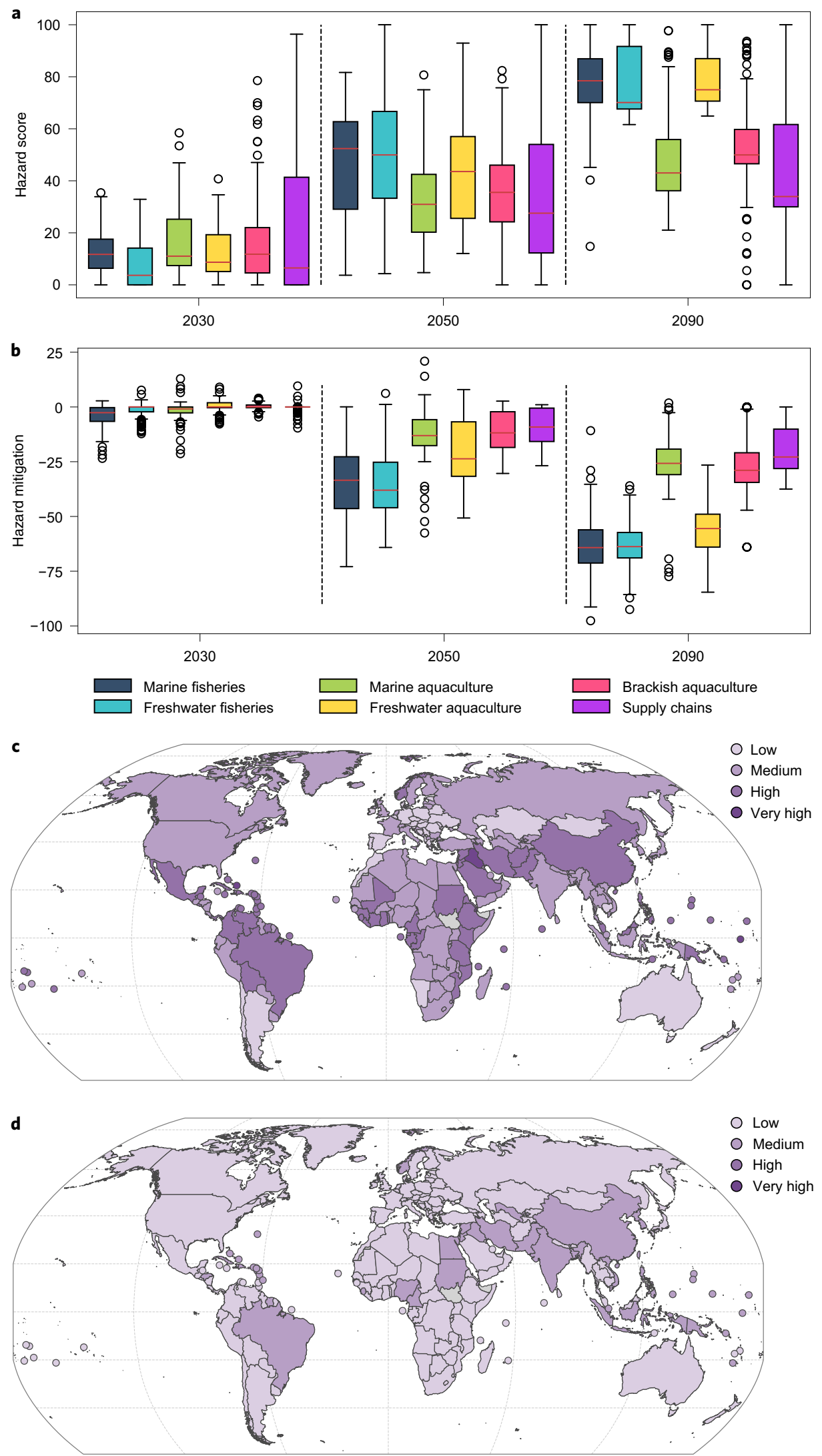

Fig. 1 Projected climate hazard scores. a, Hazard scores for each food system component for a high-emissions scenario in 2021-2040 ('2030'), 2041-2060 ('2050') and 2081-2100 ('2090'). The box limits denote the 25th and 75th percentiles, the whiskers extend to $1.5 \times$ the interquartile range from the box edges, the red line indicates the median value and the circles represent outliers. $\mathbf{b}$, Impact of emissions reduction on hazard scores for each of the components. c,d, Aggregate hazard across all components in 2050, weighted by present-day contributions, for a high-emissions scenario (c) and a low-emissions scenario (d); grey means no data. Scores <25 are classified as 'low', 25-50 as 'medium', 50-75 as 'high' and >75 as 'very high'. 
measures of exposure and vulnerability to assess the overall climate risk of aquatic foods' contributions to nutrition and health, economic, social and environmental food system outcomes for 219 countries and territories (Supplementary Methods and Supplementary Fig. 2), and we evaluated where health and sustainability are most at risk at a national level and which types of aquatic food production contribute most to that risk. On the basis of this evaluation, we identify four distinct risk profiles and corresponding policy and management actions to enhance the climate resilience of aquatic food systems.

\section{Results}

Climate impacts on aquatic food systems. Under a high-emissions, no-mitigation scenario, capture fisheries in both marine and freshwater systems are projected to face the most severe hazards (Fig. 1). By the mid-twenty-first century, marine fishery hazards are classified as 'high' (Supplementary Table 3) in most of tropical Africa, Central America and Southeast Asia (Supplementary Methods and Extended Data Fig. 1). With a larger magnitude of warming on large continental land masses than in the ocean, freshwater fisheries in some countries are projected to face 'very high' hazards (Supplementary Table 3) by the mid-twenty-first century, especially in water-stressed areas such as northern Africa and the Middle East (Extended Data Fig. 1). 'High' or 'very high' hazard scores indicate that almost all climate variables impacting these aquatic food systems are outside the range of historical variability (Supplementary Table 3).

By 2100, the estimated hazards for freshwater aquaculture under a high-emissions scenario reach the same level as those for capture-fishery systems, so that all countries face 'high' or 'very high' hazard scores for marine and freshwater fisheries and freshwater aquaculture (Fig. 1a and Extended Data Fig. 1). In contrast, marine and brackish aquaculture face lower cumulative hazards (that is, most climate variables remain within historical ranges; Supplementary Table 4) throughout the twenty-first century (Fig. 1a). Many tropical countries-where climate hazards are the strongest-do not currently practise mariculture for food, and thus skew the average hazards that marine and brackish aquaculture face downwards (Supplementary Information). As managed farming operations, (fed) marine aquaculture and brackish aquaculture are generally less directly exposed to climate-induced changes in ecosystem productivity than capture fisheries ${ }^{22}$, but they are still projected to be at risk in areas with strong impacts from sea level rise and cyclones (Extended Data Fig. 1). These types of extreme events are also important drivers of high hazards for post-harvest activities, especially in places with a large percentage of small-scale actors involved in the sector (Fig. 1a and Extended Data Fig. 1). Not included in our model but important for aquaculture are potential climate-change-induced effects on aquatic diseases and food safety risks, such as changes in harmful algal bloom occurrence ${ }^{23}$.

Strong climate mitigation efforts that are aligned with the target specified in the Paris Agreement of keeping global warming well below $2{ }^{\circ} \mathrm{C}$ can limit the hazards faced by most systems to 'medium' or 'low' (Fig. 1b). In most cases, mitigation benefits will start to materialize by the mid-twenty-first century, though certain climate variables such as sea level rise have a slow response time and will continue to impact aquatic food systems throughout the twenty-first century and beyond, even with strong near-term climate actions.
When weighted by present-day production contributions, aggregate hazards are higher in the tropics and lower across latitudes $>50^{\circ}$ (north and south) (Fig. 1c). This finding is in line with previous studies on both marine and terrestrial environments that show that the future climate will exceed the range of historical variability soonest in mid-to-low latitudes ${ }^{2,24}$. Many low-latitude communities in the least-developed or developing economies are strongly dependent-for nutrition or livelihoods or through sale to local and global markets ${ }^{6,25}$ - on the inland and marine fisheries that face the highest hazards (Extended Data Figs. 2 and 3). These least-developed or developing economies also have relatively larger social, economic and knowledge constraints (Extended Data Fig. 4) that limit them from expanding into new modes of production in the near future. For example, the development of lower-hazard offshore mariculture ${ }^{26}$ or land-based recirculating aquaculture systems ${ }^{27}$ may not be a viable climate-risk-reduction pathway for these capture-fishery-dependent countries because of the comparatively high cost and benefit concentration of these alternatives ${ }^{15,28}$. Therefore, in addition to systemic shifts in modes of production, a priority for reducing climate hazards in these economies is to reduce local hazard and exposure levels in existing production systems. Examples of such hazard reduction strategies for capture fisheries include improved water resources management, mangrove and wetland restoration ${ }^{29}$, facilitating shifts in fishing ground $\mathrm{s}^{30}$ and improved post-harvest technologies. At the same time, rapid carbon mitigation efforts by high-emitting countries can keep hazard levels to 'low' or 'medium' across production systems (Fig. 1d) and can therefore substantially reduce the risk of impacting sustainable development efforts and losing aquatic food contributions.

Compound risks to aquatic food systems. Aquatic food systems contribute comparatively more to food system outcomes in developing countries, including Small Island Developing States (SIDS), with generally higher consumption of marine and freshwater foods ${ }^{8,9}$; higher dependence on production, trade and value chain revenue $^{7,31}$; and higher numbers of aquatic food-dependent livelihoods $^{5-7}$ (Extended Data Fig. 3). Combined with the concentration of climate hazards in the tropics and subtropics (Fig. 1), this greater dependenceleads to a double jeopardy in exposure to climate hazards for nutrition and health, economic and social outcomes (Fig. 2a). Though the pattern of higher exposure to hazards in low latitudes holds true generally, national contexts play an important role in shaping differences between countries and between outcomes ${ }^{6}$. Iceland and Japan, for instance, stand out as high-latitude countries with high nutritional dependence on fish, while Brazil, as a large tropical terrestrial food producer, derives relatively low (national) nutritional and economic contributions from aquatic foods (Extended Data Fig. 3).

When each country's climate vulnerability-including economic output and inclusion, strength of social services, and metrics of food security and environmental integrity-is taken into account (Supplementary Methods and Extended Data Fig. 4), much of Africa, South and Southeast Asia, and the Indo-Pacific face 'high' to 'very high' climate risk for one or more food system outcomes by the middle of this century under a high-emissions scenario (Fig. 2b). In terms of nutrition, this may equate to reductions in aquatic food access, limiting iron, zinc, vitamin $\mathrm{B}_{12}$ and omega-3 fatty acids in

Fig. 2 | Mid-century climate risk of food system outcomes under a high-emissions scenario. a, Exposure to hazard for each food system outcome, for least-developed, developing and developed countries and SIDS ${ }^{78}$. The box limits denote the 25 th and 75 th percentiles, the whiskers extend to $1.5 \times$ the interquartile range from the box edges, the red line indicates the median value and the circles represent outliers. $\mathbf{b}$, Maximum risk score across all outcomes. c, Number of outcomes for which the risk score is 'high' or 'very high'. d, Number of countries for which the risk score is 'high' (light shade) or 'very high' (dark shade) for a low-emissions scenario (yellow) and a high-emissions scenario (blue) in 2030, 2050 and 2090. Grey means no data. Scores $<25$ are classified as 'low', 25-50 as 'medium', 50-75 as 'high' and $>75$ as 'very high'. 
populations that are already at thresholds for deficiency ${ }^{9}$, especially if climate change drives up food prices $^{32}$ for households least likely to afford fish. In contrast, most countries in North America and
Europe, as well as Chile, Argentina, Australia and New Zealand, face 'low' to 'medium' climate risk across all food system outcomes for this period (Fig. 2c).
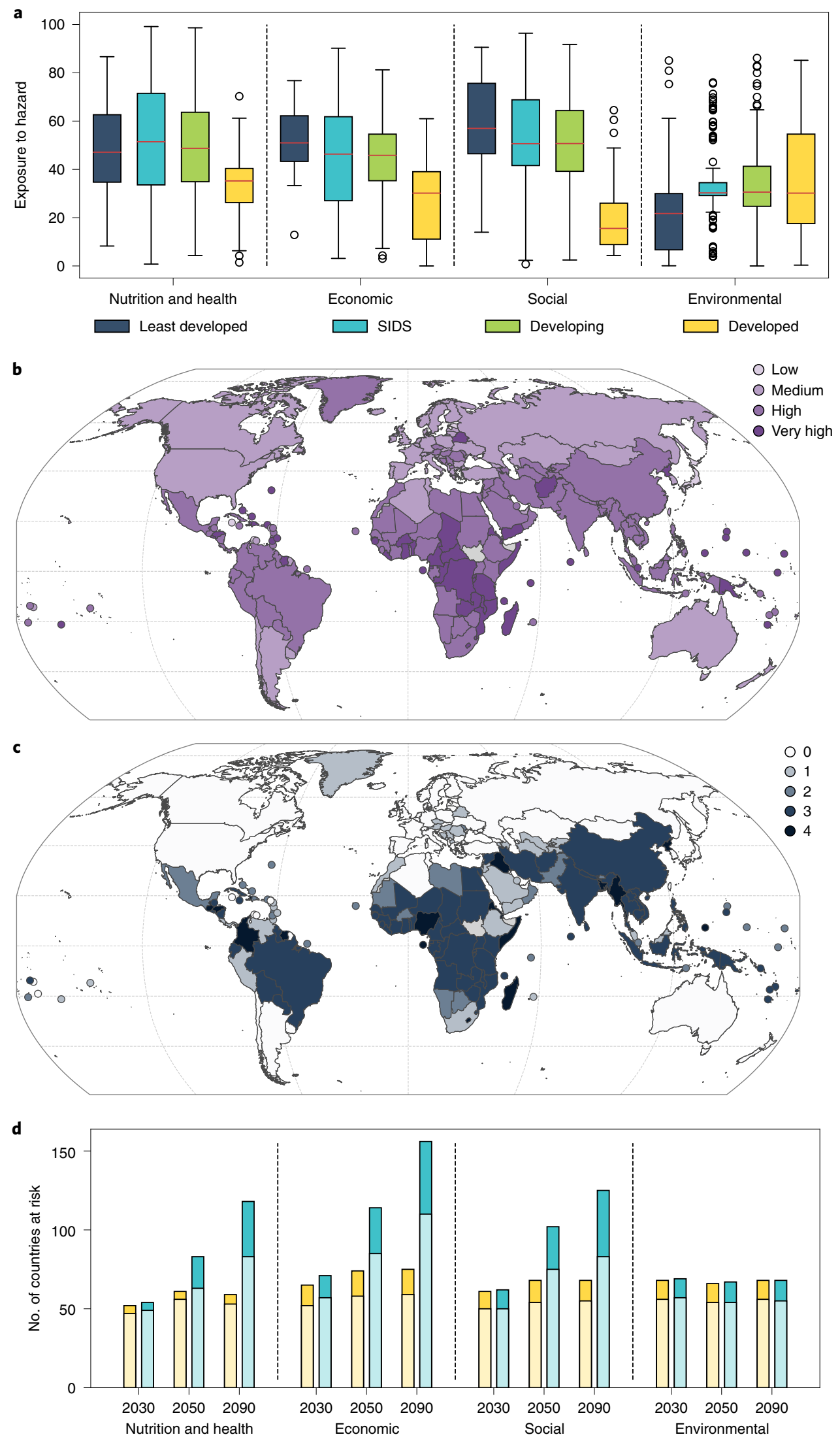


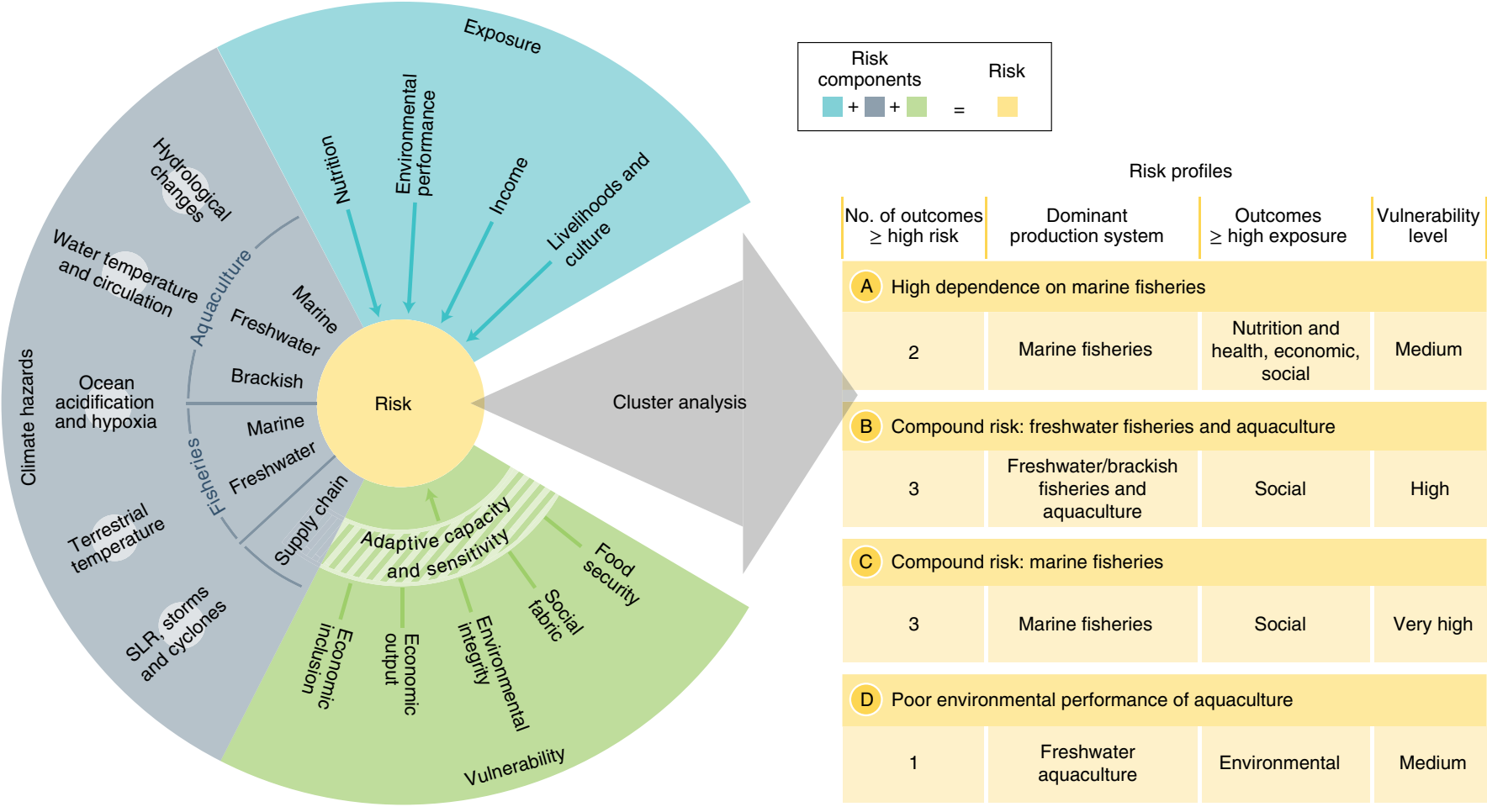

Fig. 3 | Climate risk profiles based on differences in hazards, exposure and vulnerability across food system outcomes. On the basis of a cluster analysis (Supplementary Information), we identify four climate risk profiles for aquatic food system outcomes. The profiles are based on the characteristics of countries that have 'high' or 'very high' climate risk for at least one food system outcome by mid-century under a high-emissions scenario. The profile descriptions are based on median indicator values for each profile (Extended Data Fig. 7). Individual countries within each cluster can deviate slightly from this characterization. SLR, sea level rise.

A rapid reduction in carbon emissions can avoid substantial increases in climate risk for many countries by the end of this century (Extended Data Figs. 5 and 6). Under a high-emissions scenario, the number of countries facing 'high' risk for nutrition and health, economic and social outcomes increases from about 50 to over 80 between the near future and the late twenty-first century, and those facing 'very high' risk increase from about 10 to about 40. Such an increase is largely prevented under a low-emissions scenario (Fig. 2d). Still, due to high vulnerability and aquatic-food dependence, at least 50 countries will experience 'high' or 'very high' risk throughout the twenty-first century in all carbon emission scenarios. Our findings thus show that multiple types of risk need attention through policy and investments (see next section) to ensure that beneficial aquatic food system outcomes are secured for the most vulnerable countries.

A cluster analysis of our modelling results (Supplementary Information) delineates four distinct climate risk profiles for countries that experience high risk in at least one food system outcome under high emissions by the mid-twenty-first century (Figs. $2 \mathrm{~b}$ and 3, Table 1, and Extended Data Figs. 7 and 8). For many coastal countries and SIDS with high dependency on the marine fishery sector and medium vulnerability, climate risks are concentrated in the nutrition and health and/or economic food system outcomes. Similarly, some countries, primarily in Eastern Europe, generally face low hazards and display medium vulnerability but stand out for their poor environmental performance ${ }^{33}$, leading to concentrated climate risks in the environmental dimension of food system outcomes. Countries with high climate vulnerability are projected to face compound climate risks ${ }^{34,35}$ across three or four of the food system outcomes, either in marine fisheries (primarily coastal Africa) or in freshwater and deltaic fisheries and aquaculture (South and Southeast Asia and Central Africa). These different risk profiles across countries and regions call for region-specific and context-specific risk reduction interventions.

Towards climate-resilient aquatic foods. The high levels of climate risk for multiple outcomes across much of the developing world (Fig. 2) call for urgent action to support the long-term sustainability $^{14}$, resilience ${ }^{36}$ and equity ${ }^{13,15}$ of aquatic food systems. Enhanced resilience could, in principle, be achieved by the implementation of adaptive and/or transformative measures at any point along the risk chain, including reducing the actual climate hazards (for example, greenhouse gas emissions reductions), reducing the sensitivity of the production systems to these hazards (for example, farming climate-tolerant species with reduced feed dependence ${ }^{27,37}$, and building barriers and restoring coastal ecosystems to protect against storm $\mathrm{s}^{27}$ ), reducing dependence on climate-sensitive aquatic foods and sectors (for example, diet and livelihood diversification ${ }^{38}$ ), and reducing vulnerability through investments that benefit human development irrespective of climate change (for example, human capabilities investments, including gender consideration $\mathrm{s}^{39}$ ).

Climate mitigation activities will substantially reduce climate hazards (Fig. 1) but are insufficient to avoid negative aquatic food system outcomes, as a certain degree of climate change is unavoidable (Fig. 3). A sensitivity analysis on the vulnerability variables in our model (Supplementary Information) shows that reducing vulnerability results in similar levels of climate change risk reduction to meeting mitigation targets in the Paris Agreement, especially for countries that face compound climate risks (such as Malawi and El Salvador; Supplementary Table 12). These findings highlight the need to focus on the broader social-ecological context of each country, with actions building resilience that extend beyond aquatic food production ${ }^{40}$. Priority strategies for building resilience, as well as policy and management considerations and potential trade-offs, 
Table 1 | Climate resilience priorities, policy and management considerations, and trade-offs for four climate risk profiles

Resilience priorities

Policy and management considerations
Potential trade-offs for health,

sustainability and equity

(A) High nutritional, economic and social dependency on marine fisheries (for example, Ghana, Palau, Peru, Timor-Leste and Viet Nam)

Secure sustainable marine fishing practices and policies, ensure that benefits flow to vulnerable groups, and develop alternatives that increase adaptive capacity.
$P$ : Shift to offshore resources; climate-smart and climate-just agreements for transboundary resources; policies to enable the utilization of nutrient-rich species in coastal communities. F\&l: Conservation financing for small-scale fisheries; revised fishery subsidies to incentivize sustainability.

T\&l: Local adaptation measures for fisheries (for example, development of national pelagic fisheries); climate-informed and equitable vessel scheme days.

B\&S: Equitable and climate-responsive access agreements; export policies that ensure sufficient domestic supply.
- Nutritional content, economic value and stock sustainability of alternative species.

- Local nutrition needs versus revenue generation from exports; revenue versus livelihoods.

- Gender dimension of livelihood opportunities.

(B) Compound climate risk-freshwater/deltaic fisheries and aquaculture (for example, Bangladesh, Cambodia, Central African Republic and Malawi)

Sustain threatened freshwaters while integrating climate-adaptive and sustainable aquatic food systems into socio-economic development efforts.
$P$ : Protection and management of freshwater fisheries and wetland ecosystems; inclusion of small-scale fisheries in fisheries policies. F\&l: Investment in the protection and management of natural capital; alternative livelihoods; collective loans and savings programmes. T\&I: Freshwater aquaculture; integration of fish into water management structures, planning and management; sustainable intensification. B\&S: Fair trade agreements for the benefits of local people (including intergenerational discounting); climate information services and early warning systems.
- Immediate needs and disaster risk reduction versus long-term adaptation and sustainability interventions.

- Competition over freshwater resources.

- Export versus local needs.

- Fish as food versus feed.

- Equity and gender dimensions of adaptive programming activities.

- Immediate needs and disaster risk reduction versus long-term adaptation and sustainability interventions.

- Export versus local needs.

- Equity and gender dimensions of adaptive programming activities. development efforts. and engages marginalized groups.

F\&l: Financial incentives for fishers (credit support, minimum supportive price and so on); financial management through collectives and cooperatives; gender transformative adaptation opportunities. T\&I: Infrastructure development, including technology such as cold storage.

B\&S: Equitable fisheries access agreements; climate information services and early warning systems.

(D) Environmental performance risk of freshwater aquaculture (for example, Belarus, Bosnia and Herzegovina, and Israel)

Improve environmental performance of aquatic food production systems
P: Environmental regulations; aquaculture zoning; limitations on carrying • capacity.

F\&l: Financing options for supply chain actors transitioning to low-impact practices.

T\&I: Solar energy; water-efficient systems; reductions in waste and losses along value chains; production optimization, including sustainable intensification.

B\&S: Incentivization schemes for sustainable farming practices (for example, certification).

We provide examples of high-level policy and management considerations in the following categories: policies (P), finance and investment (F\&I), technology and innovation (T\&I), and business and services $(B \& S)$. Resilience-enhancing activities are not exclusive to any particular risk profile, and knowledge exchange can occur both within and across profiles. The profile letters A-D correspond to the risk profiles in Fig. 3. References supporting particular policy and management considerations are listed in the Supplementary Information.

differ between countries belonging to each of our identified risk profiles (Table 1).

Countries with low to medium vulnerability (the 'high dependence on marine fisheries' and 'poor environmental performance of aquaculture' profiles; Fig. 3) tend to experience high climate risk for just one or two food system outcomes, meaning that targeted interventions in specific areas can reduce risk (Table 1). For countries with high marine fisheries dependence, including many SIDS, one of the challenges will be to design measures that strike an appropriate balance between supporting economic development aspirations through efficiency and revenue generation, and supporting food security through local and domestic consumption of fish ${ }^{41,42}$ (for example, climate-smart agreements for transboundary resources ${ }^{43}$ and the development of climate-resilient aquaculture for food secu$\left.r^{2} y^{44}\right)$. For countries where freshwater aquaculture contributes to poor environmental outcomes, solutions may target the adoption of integrated farming solutions or of technological innovations such as resource-efficient production systems that can be isolated from the environment ${ }^{27,37}$. In both contexts, solutions need support through enabling government policies, functional institutions at the national to community levels and sustainable, responsible financial investment ${ }^{44}$.

Enhancing climate resilience for highly vulnerable countries facing compound climate risk (Table 1 and Extended Data Fig. 8) - from freshwater and deltaic fisheries and aquaculture or from marine fisheries (Fig. 3) -is most challenging and urgent given that these countries are projected to have the greatest number of food system outcomes experiencing high climate risk. For such countries, resilience efforts focused on aquatic food systems provide options (such as nature-based solutions (for example, mangrove, reef and 
seagrass restoration to aid coastal storm protection and enhance aquatic ecosystem productivity) ${ }^{29}$, sustainable intensification ${ }^{45}$, livelihood diversification ${ }^{38}$ and investments in local value chains ${ }^{44}$ ), but these efforts need to be part of a more generalized resilience framework that addresses the social dimensions of vulnerability ${ }^{39,40,46,47}$ (for example, through strengthening governance, promoting gender equity and reducing poverty; Supplementary Table 12). It is worth noting that 'no-regret' investments ${ }^{39}$ based on net social welfare gain have proven a challenge in practice-particularly where investments fail to deliver a 'net political gain', as determined by a set of complex value preferences. Climate solutions that require public sector investments must be able to deliver both social and political gains to increase their acceptability to the public choice-maker. Ultimately, a generalized resilience approach means enhancing the capacity of coastal and riparian people to become the agents of societally desired systems transformation ${ }^{40,47}$ and to recognize aquatic food systems as integral to socio-economic development efforts and nutrition policies ${ }^{42}$ and overall food system resilience ${ }^{48,49}$.

\section{Discussion}

The availability of appropriate data and modelling tools for better understanding risks and resilience priorities at a global scale poses a challenge, especially for countries and systems considered most at risk. For example, in calculating hazard scores for freshwater systems and supply chains, we were unable to assess changes in climate variables at a subnational scale or to consider the varying sensitivities of different ecosystems or production types and post-production processes $^{1,17,49}$. For many SIDS, key indicators were missing from global databases (Supplementary Information), precluding holistic climate risk assessment ${ }^{50}$. The risk of countries such as Bangladesh, Cambodia and the countries of the African Great Lakes region (which are among the largest freshwater-fish-producing nations in the world) may in reality be even higher given that inland fish production is substantially underestimated in many countries ${ }^{25,51}$. Failing to address these gaps in data and understanding can perpetuate inequities, as resilience investments are likely to go to places and systems already assessed in the research and policy literature ${ }^{13}$.

Our analysis assumed that exposure and vulnerability remain unchanged in time, though different development trajectories could change climate risk in magnitudes commensurate with changes driven by different emission trajectories (as shown by our sensitivity analysis; Supplementary Table 12). Future work could build on the framework developed here to explore the impacts of various socio-economic pathways ${ }^{52}$ and their interactions with climate hazards. Similarly, climate change will also impact food systems outside the aquatic realm, with unknown implications for feedbacks, trade-offs, relative risk and adaptive options. While this research expands our understanding of the combined and comparative climate risk of all aquatic food systems, we ultimately need a holistic climate risk framework that makes our entire plate (staples, nuts and legumes, fruits and vegetables, livestock, and aquatic foods) resilient ${ }^{21}$.

Through an integrative climate and aquatic food systems modelling approach, we reveal four main climate risk profiles and identify key challenges and opportunities for building pathways towards climate-resilient aquatic food systems. Of the different environments in which aquatic foods are caught and produced, fisheries and freshwater aquaculture are projected to experience the highest cumulative hazards throughout the twenty-first century. Combined with a comparatively higher dependence on aquatic foods for nutrition, income and livelihoods-and greater vulnerability to the loss of these benefits-SIDS and countries in Africa, South Asia and Southeast Asia are projected to be at particularly high risk from climate change.

Differences in climate risk profiles call for resilience-enhancing actions that are region-specific and context-specific and are guided by principles of equity and fairness ${ }^{13,15}$. Most urgent are actions and investments for countries that face compound climate risk across aquatic food system outcomes, requiring transformative change to reduce societal vulnerability. Though we focused on countries with high climate risk, countries with low to medium risk will also face increasing climate hazards domestically and are connected to transboundary climate risk through species movement and trade of aquatic foods and inputs ${ }^{43,53}$. This calls for justice-informed collaboration towards climate-proofing of aquatic food systems that transcends national and regional boundaries.

\section{Methods}

Assessment of climate impact pathways. We reviewed literature covering a wide range of geographic areas-from global reviews to regional and national case studies-to identify climate pathways that impact aquatic food systems in three different capacities: (1) aquatic food production systems, (2) aquatic food supply chains and (3) aquatic food system outcomes. In our review, we first focused on large assessment reports such as those produced by the Intergovernmental Panel on Climate Change ${ }^{2}$, followed by comprehensive scientific reviews and meta-analyses (for example, refs. ${ }^{1,4,17,54,55}$ ) and the papers cited therein. We filled in remaining gaps using strategic keyword searches and the expert knowledge of our multidisciplinary team of co-authors. An overview and ranking of climate pathways for aquatic food production systems and supply chains, including key references, can be found in Supplementary Table 11, with a brief narrative summary in the Supplementary Methods.

Aquatic food production systems were split into capture fisheries and aquaculture and were further grouped by marine and freshwater production environments. The aquatic food supply chain was divided into seven components (input supply, production, post-harvest storage, processing, distribution, marketing and retail, and consumption and utilization ${ }^{11}$ ), though most literature addressed only the production stage. For the different production systems, we ranked the impact of each climate change pathway by direction (positive impact, negative impact or varied impact) and degree of confidence (limited, medium or robust research). For the supply chain components, we just noted the presence or absence of an impact.

Hazard, exposure and vulnerability variables. We compute quantitative indices of climate risk for the four aquatic food system outcomes-nutrition and health, economic, social, and environmental-adopting a fuzzy logic modelling approach to implement the risk assessment framework used by the Intergovernmental Panel on Climate Change ${ }^{2}$. In this framework, climate risk results from the interaction between climate-change-induced hazards, exposure to those climate hazards and vulnerabilities of components of the aquatic food systems. For our purposes, we conceptualize climate hazards as the dominant climate variables that impact aquatic food production and supply chains, exposure as the degree to which aquatic foods contribute to the various food system outcomes at a national level, and vulnerability as a combination of sensitivity to and adaptive capacity of the nationally aggregated food systems in the face of the loss of aquatic food contributions. Through two rounds of virtual workshops, our team of co-authors (selected for their expertise spanning marine and freshwater ecosystems, fisheries and aquaculture production systems, and multiple food system outcomes) selected hazard, exposure and vulnerability indicators on the basis of their expert knowledge, published literature and data availability for most of the 219 countries included in this study.

Climate hazards. From our literature assessment (Supplementary Table 11), we identified the most impactful and well-understood climatic drivers of changes in aquatic food production for which data and/or modelling tools are available at a global level. These drivers include changes in ocean temperature, circulation, net primary productivity and dissolved oxygen concentrations, which together are used to calculate projected changes in marine fisheries catch and aquaculture yield using a dynamic biological envelope $\operatorname{model}^{56}$. Additionally, changes in ocean $\mathrm{pH}$, the extent of sea ice and the cumulative mean intensity of marine heatwaves ${ }^{57}$ are used as marine hazard variables. Inundation from projected sea level rise and the strength of tropical storms threaten marine and brackish aquaculture as well as supply chain processes. For freshwater systems, near-surface $(2 \mathrm{~m})$ air temperature changes over land and changes in precipitation are used. Finally, projected changes in fish meal availability and global crop land temperature capture the hazards (through cost of production) to feed-dependent aquaculture production. Some climate impact pathways, such as harmful algal blooms and their impacts on food safety, are known to be important but were excluded because (to our knowledge) no appropriate and published global model or dataset of national-level indicator variables is yet available. An overview of all hazard variables used in the model is presented in Supplementary Table 6.

To represent uncertainties in projections of environmental changes by different ESMs, where possible, we used projections from three ESMs available from CMIP6 (ref. ${ }^{58}$ ): Geophysical Fluid Dynamics Laboratory (GFDL)-ESM4 (ref. ${ }^{59}$ ), 
the Institut Pierre-Simon Laplace (IPSL)-CM6A-LR ${ }^{60}$ and Max Planck Institute (MPI)-ESM1-2-HR ${ }^{61}$. Outputs from GFDL-ESM4 and IPSL-CM6A-LR are also used by the Fisheries and Marine Ecosystem Impact Models Intercomparison Projects, while MPI-ESM1-2-HR is used additionally by the Dynamic Bioclimate Envelope Model to project future changes in maximum catch potential. We calculated climate hazards using two contrasting scenarios: Shared Socio-economic Pathway (SSP) 1-Representative Concentration Pathway (RCP) 2.6 (SSP1-2.6) and SSP5-8.5. SSP1-2.6 and SSP5-8.5 represent a 'strong mitigation' low-emissions pathway and a 'no mitigation' high-emissions pathway, respectively ${ }^{62}$. For the marine heatwave variable, CMIP6 results were not yet available, so CMIP5 equivalents were used ${ }^{57}$

Results are presented for the near future (2021-2040) and the middle (2041$2060)$ and end (2081-2100) of the twenty-first century. As we compute climate risk indices for 219 countries and territories, we focus on hazards that are most common across these geographies and for which global datasets are available. Some of the climate hazards (such as the impacts of harmful algal blooms and the spread of disease) are indirectly represented by environmental variables that drive these hazards (such as ocean temperature and dissolved oxygen level). While our modelling approach accounts for interactions between climate hazards in some components of the aquatic food systems (for example, marine capture fisheries and aquaculture production), the interacting effects of hazards on some other components are not well understood, so these are not explicitly represented in the risk assessment model.

Exposure. The exposure of countries or territories to climatic hazards through their aquatic food systems is positively associated with their level of dependency on aquatic foods to support nature and people. We selected indicators (Supplementary Table 7) for four food system outcomes: nutrition and health, economic, social and environment. For nutrition and health outcomes, the indicators are per capita supply of marine and freshwater aquatic foods and the percentage of a nation's consumption of vitamin $B_{12}$ and DHA + EPA fatty acids (types of omega- 3 fatty acids specific to aquatic foods) that is derived from aquatic foods ${ }^{9}$. For economic outcomes, we use data on the contributions of aquatic food to gross domestic product (GDP) $25,31,63,64$, estimates of economic multipliers of marine supply chains ${ }^{65}$ and net aquatic food trade balance relative to $\mathrm{GDP}^{66}$. As social outcome variables, we selected the contributions of marine fisheries, aquaculture and inland fisheries to employment ${ }^{5,25,67}$, as well as the ratio of indigenous to national-average consumption of seafood as a metric of the cultural importance of aquatic foods ${ }^{68}$. Finally, the environmental outcome variables are drawn from standardized estimates of the average greenhouse gas emissions, nitrogen and phosphorus emissions, and land use and freshwater use of different types of wild-capture and farmed aquatic food production ${ }^{33}$.

Vulnerability. To capture the vulnerability of aquatic food system outcomes to climate hazards, we selected several variables (Supplementary Table 8) that represent the adaptive capacity of aquatic food systems and the sensitivity of aquatic food system outcomes in the face of climate change. As more generalized metrics of adaptive capacity and sensitivity, we include GDP per capita ${ }^{63}$, an aggregate of the Worldwide Governance Indicators ${ }^{69}$, educational attainment ${ }^{70}$, percentage of the population below the national poverty line ${ }^{63}$, the Gini coefficient ${ }^{63}$, access to financial services ${ }^{71}$, research and development expenditure as a percentage of $\mathrm{GDP}^{63}$, and the percentage of aquatic food landings from small-scale operations $s^{72}$. As outcome-specific metrics, we include the stunting rate of children under five ${ }^{73}$ and summary exposure values for vitamin $\mathrm{B}_{12}$ and $\mathrm{DHA}+\mathrm{EPA}$ fatty acids ${ }^{9}$ for nutrition and health outcomes, and the Biodiversity and Habitat, Fisheries, and Climate Change indicators of the Environmental Performance Index ${ }^{74}$ for environmental outcomes.

A more detailed accounting of each of the model variables, including justification and data processing steps as well as a statistical summary, can be found in the Supplementary Methods. For each of the risk components, scores were calculated using whichever of the indicator variables were available.

Fuzzy logic system. We apply a fuzzy logic modelling approach to compute climate risks and account for the inherent data and knowledge uncertainties present in determining climate risks of national food system outcomes. Such an approach has been previously applied to study the conservation risk of marine fish stocks from fishing ${ }^{20}$ and climate change $\mathrm{f}^{75,76}$. In brief, the fuzzy logic algorithm is divided into three steps ${ }^{20}$ :

1. Fuzzification: Indicator values are categorized into one or more levels of 'low', 'medium, 'high' and 'very high' (hazard, exposure and vulnerability) simultaneously, with the degree of membership in each level defined by fuzzy membership functions (Supplementary Fig. 1 and Supplementary Table 9).

2. Fuzzy reasoning: For each subcomponent of climate risk (hazard, exposure and vulnerability), the degree of membership associated with each level is cumulated using an algorithm called MYCIN $^{20}$ :

$$
\operatorname{AccMem}_{i+1}=\operatorname{AccMem}_{i}+\text { Membership }_{i+1} \times\left(1-\operatorname{AccMem}_{i}\right)
$$

where AccMem is the accumulated membership of a particular level (for example, high vulnerability) and $i$ denotes the indicator variable contributing to the subcomponent. Then, hazard, exposure and vulnerability are aggregated into a combined risk level using predefined heuristic rules (Supplementary Table 2). Where data availability allows, information is kept segregated on the basis of the relevant subsystem (such as fisheries versus aquaculture or marine versus freshwater)

3. Defuzzification: The climate risk of aquatic food system outcomes is ultimately expressed on a scale of 0 to 100 , with 100 being the most at risk. The index values (Indval) corresponding to each level $x$ are: 'low' $=1$, 'moderate' $=25$, 'high' $=75$ and 'very high' $=100$. The final risk index (FnlInd) is calculated from the average of the index values, weighted by their accumulated membership ${ }^{20}$ :

$$
\text { FnlInd }=\frac{\sum_{x=1}^{4} \operatorname{AccMem}_{x} \times \operatorname{Indval}_{x}}{\sum_{x=1}^{4} \operatorname{AccMem}_{x}}
$$

A more detailed description of the various fuzzy logic modelling steps and assumptions and a schematic overview of the various model components are provided in the Supplementary Information.

Cluster analysis. To identify patterns in the types of climate risk that high-risk countries face, we perform a $K$-means clustering analysis on the outcomes of the fuzzy logic model, using the following input variables: the number of outcomes for which climate risk in 2050 is 'high' or 'very high' under SSP5-8.5, the climate hazard score in 2050 under SSP5-8.5, the percentage of production from marine environments $^{64}$, the percentage of production from aquaculture ${ }^{64}$, exposure scores for each of the food system outcomes (nutrition and health, economic, social, and environmental) and vulnerability scores for each of the food system outcomes. Only countries for which at least one of the food system outcomes had 'high' or 'very high' risk in 2050 under SSP5-8.5 were included. We were not able to assign clusters for countries with missing values for any of these variables.

We find that using a total of four clusters leads to climate risk profiles that are distinct (Supplementary Fig. 3) and meaningful for describing policy considerations. The profiles that we identify are high dependency on marine fisheries (Cluster 2, 47 countries), compound climate risk from freshwater and brackish systems (Cluster 1, 38 countries), compound climate risk from marine fisheries (Cluster 3, 28 countries), and environmental performance risk from freshwater aquaculture (Cluster 4, 17 countries). The distribution of input variables for each of these clusters is shown in Extended Data Fig. 7 and summarized in Supplementary Table 13. A map of the cluster assignments is shown in Extended Data Fig. 8, with a complete list in Supplementary Table 14.

Statistics and reproducibility. No data were excluded from the analyses.

\section{Data availability}

Most of the model input data were retrieved from publicly accessible reports and databases, as outlined in Supplementary Tables 6-8. The model output data are provided on Dryad ${ }^{77}$ : https://doi.org/10.5061/dryad.70rxwdbz3. Source data are provided with this paper.

\section{Code availability}

All custom code produced for the analyses was generated using Python version 3.7.1 and $\mathrm{R}$ version 4.0.2 and is available from the corresponding author upon request.

Received: 23 March 2021; Accepted: 17 August 2021; Published online: 15 September 2021

\section{References}

1. Impacts of Climate Change on Fisheries and Aquaculture: Synthesis of Current Knowledge, Adaptation and Mitigation Options Vol. 627 (FAO, 2019).

2. IPCC Special Report on the Ocean and Cryosphere in a Changing Climate (eds Pörtner, H.-O. et al.) (IPCC, 2019).

3. Fanzo, J., Davis, C., McLaren, R. \& Choufani, J. The effect of climate change across food systems: implications for nutrition outcomes. Glob. Food Sec. 18, 12-19 (2018)

4. Myers, S. S. et al. Climate change and global food systems: potential impacts on food security and undernutrition. Annu. Rev. Public Health 38, 259-277 (2017)

5. Teh, L. C. L. \& Sumaila, U. R. Contribution of marine fisheries to worldwide employment: global marine fisheries employment. Fish Fish. 14, 77-88 (2013).

6. Selig, E. R. et al. Mapping global human dependence on marine ecosystems. Conserv. Lett. 309, e12617 (2018).

7. The State of World Fisheries and Aquaculture 2020: Sustainability in Action (FAO, 2020); https://doi.org/10.4060/ca9229en

8. Bennett, A. et al. Contribution of Fisheries to Food and Nutrition Security: Current Knowledge, Policy, and Research (Duke University, 2018). 
9. Golden, C. D. et al. Aquatic foods to nourish nations. Nature (in the press).

10. Cheung, W. W. L. et al. Large-scale redistribution of maximum fisheries catch potential in the global ocean under climate change. Glob. Change Biol. 16, 24-35 (2010).

11. Nutrition and Food Systems (HLPE, 2017).

12. Hicks, C. C. et al. Harnessing global fisheries to tackle micronutrient deficiencies. Nature 574, 95-98 (2019).

13. Österblom, H. et al. Towards Ocean Equity (High Level Panel for a Sustainable Ocean Economy, 2020); https://digitalarchive.worldfishcenter.org/ bitstream/handle/20.500.12348/4486/71d48a67e55853a80e461c0ba5529caf.pdf

14. Sustainable Fisheries and Aquaculture for Food Security and Nutrition (HLPE, 2014); http://www.fao.org/3/a-i3844e.pdf

15. Farmery, A. K. et al. Blind spots in visions of a 'blue economy' could undermine the ocean's contribution to eliminating hunger and malnutrition. One Earth 4, 28-38 (2021).

16. Transforming Our World: The 2030 Agenda for Sustainable Development (United Nations, 2015).

17. Reid, G. K. et al. Climate change and aquaculture: considering biological response and resources. Aquac. Environ. Interact. 11, 569-602 (2019).

18. Myers, B. J. E. et al. Global synthesis of the documented and projected effects of climate change on inland fishes. Rev. Fish Biol. Fish. 27, 339-361 (2017).

19. Froehlich, H. E., Runge, C. A., Gentry, R. R., Gaines, S. D. \& Halpern, B. S. Comparative terrestrial feed and land use of an aquaculture-dominant world. Proc. Natl Acad. Sci. USA 115, 5295-5300 (2018).

20. Cheung, W. W. L., Pitcher, T. J. \& Pauly, D. A fuzzy logic expert system to estimate intrinsic extinction vulnerabilities of marine fishes to fishing. Biol. Conserv. 124, 97-111 (2005).

21. Halpern, B. S. et al. Opinion: putting all foods on the same table: achieving sustainable food systems requires full accounting. Proc. Natl Acad. Sci. USA 116, 18152-18156 (2019).

22. Froehlich, H. E., Gentry, R. R. \& Halpern, B. S. Global change in marine aquaculture production potential under climate change. Nat. Ecol. Evol. 2, 1745-1750 (2018).

23. Brown, A. R. et al. Assessing risks and mitigating impacts of harmful algal blooms on mariculture and marine fisheries. Rev. Aquac. https://doi.org/ 10.1111/raq.12403 (2019).

24. Battisti, D. S. \& Naylor, R. L. Historical warnings of future food insecurity with unprecedented seasonal heat. Science 323, 240-244 (2009).

25. Funge-Smith, S. Review of the State of the World Fishery Resources: Inland Fisheries (FAO, 2018); http://www.fao.org/3/ca0388en/CA0388EN.pdf

26. Gentry, R. R. et al. Mapping the global potential for marine aquaculture. Nat. Ecol. Evol. 1, 1317-1324 (2017).

27. Reid, G. K. et al. Climate change and aquaculture: considering adaptation potential. Aquac. Environ. Interact. 11, 603-624 (2019).

28. Belton, B. et al. Farming fish in the sea will not nourish the world. Nat. Commun. 11, 5804 (2020).

29. Gattuso, J.-P. et al. Ocean solutions to address climate change and its effects on marine ecosystems. Front. Mar. Sci. 5, 337 (2018).

30. Ojea, E., Lester, S. E. \& Salgueiro-Otero, D. Adaptation of fishing communities to climate-driven shifts in target species. One Earth 2, 544-556 (2020).

31. Hidden Harvest: The Global Contribution of Capture Fisheries (World Bank, 2012); http://documents1.worldbank.org/curated/en/515701468152718292/pdf /664690ESW0P1210120HiddenHarvest0web.pdf

32. Tigchelaar, M., Battisti, D. S., Naylor, R. L. \& Ray, D. K. Future warming increases probability of globally synchronized maize production shocks. Proc. Natl Acad. Sci. USA 115, 6644-6649 (2018).

33. Gephart, J. A. et al. Environmental performance of blue foods. Nature (in the press).

34. Phillips, C. A. et al. Compound climate risks in the COVID-19 pandemic. Nat. Clim. Change 10, 586-588 (2020).

35. Cutter, S. L. The changing nature of hazard and disaster risk in the Anthropocene. Ann. Assoc. Am. Geogr. 111, 819-827 (2021).

36. Tendall, D. M. et al. Food system resilience: defining the concept. Glob. Food Sec. 6, 17-23 (2015)

37. Lebel, L. et al. Innovation, practice, and adaptation to climate in the aquaculture sector. Rev. Fish. Sci. Aquac. https://doi.org/10.1080/23308249.202 0.1869695 (2021)

38. Jørstad, H. \& Webersik, C. Vulnerability to climate change and adaptation strategies of local communities in Malawi: experiences of women fish-processing groups in the Lake Chilwa Basin. Earth Syst. Dyn. 7, 977-989 (2016).

39. Heltberg, R., Siegel, P. B. \& Jorgensen, S. L. Addressing human vulnerability to climate change: toward a 'no-regrets' approach. Glob. Environ. Change 19, 89-99 (2009).

40. Cinner, J. E. et al. Building adaptive capacity to climate change in tropical coastal communities. Nat. Clim. Change 8, 117-123 (2018).
41. Bell, J. D. et al. Diversifying the use of tuna to improve food security and public health in Pacific Island countries and territories. Mar. Policy 51, 584-591 (2015).

42. Bennett, A. et al. Recognize fish as food in policy discourse and development funding. Ambio https://doi.org/10.1007/s13280-020-01451-4 (2021).

43. Oremus, K. L. et al. Governance challenges for tropical nations losing fish species due to climate change. Nat. Sustain. 3, 277-280 (2020).

44. Roscher, M. et al. Building Adaptive Capacity to Climate Change: Approaches Applied in Five Diverse Fisheries Settings (CGIAR, 2018); https://digitalarchive. worldfishcenter.org/bitstream/handle/20.500.12348/2094/FISH-2018-18. pdf? sequence $=2$ \&isAllowed $=y$

45. Belton, B., Reardon, T. \& Zilberman, D. Sustainable commoditization of seafood. Nat. Sustain. https://doi.org/10.1038/s41893-020-0540-7 (2020).

46. Thomas, K. et al. Explaining differential vulnerability to climate change: a social science review. WIREs Clim. Change 10, e565 (2019).

47. Adger, W. N., Brown, K., Butler, C. \& Quinn, T. Social ecological dynamics of catchment resilience. Water 13, 349 (2021).

48. Troell, M. et al. Does aquaculture add resilience to the global food system? Proc. Natl Acad. Sci. USA 111, 13257-13263 (2014).

49. Davis, K. F., Downs, S. \& Gephart, J. A. Towards food supply chain resilience to environmental shocks. Nat. Food 2, 54-65 (2021).

50. Monnereau, I. et al. The impact of methodological choices on the outcome of national-level climate change vulnerability assessments: an example from the global fisheries sector. Fish Fish. 18, 717-731 (2017).

51. Fluet-Chouinard, E., Funge-Smith, S. \& McIntyre, P. B. Global hidden harvest of freshwater fish revealed by household surveys. Proc. Natl Acad. Sci. USA 115, 7623-7628 (2018).

52. Maury, O. et al. From shared socio-economic pathways (SSPs) to oceanic system pathways (OSPs): building policy-relevant scenarios for global oceanic ecosystems and fisheries. Glob. Environ. Change 45, 203-216 (2017).

53. Gephart, J. A., Rovenskaya, E., Dieckmann, U., Pace, M. L. \& Brännström, Å. Vulnerability to shocks in the global seafood trade network. Environ. Res. Lett. 11, 035008 (2016).

54. Daw, T., Adger, W. N., Brown, K. \& Badjeck, M.-C. in Climate Change Implications for Fisheries and Aquaculture: Overview of Current Scientific Knowledge (eds Cochrane, K. et al.) 107-150 (FAO, 2009).

55. Gaines, S. et al. The Expected Impacts of Climate Change on the Ocean Economy (World Resources Institute, 2019).

56. Cheung, W. W. L. et al. Structural uncertainty in projecting global fisheries catches under climate change. Ecol. Modell. 325, 57-66 (2016).

57. Frölicher, T. L., Fischer, E. M. \& Gruber, N. Marine heatwaves under global warming. Nature 560, 360-364 (2018).

58. Eyring, V. et al. Overview of the Coupled Model Intercomparison Project Phase 6 (CMIP6) experimental design and organization. Geosci. Model Dev. 9, 1937-1958 (2016).

59. Dunne, J. P. et al. The GFDL Earth System Model version 4.1 (GFDL-ESM 4.1): overall coupled model description and simulation characteristics. J. Adv. Model. Earth Syst. 12, e2019MS002015 (2020).

60. Boucher, O. et al. Presentation and evaluation of the IPSL-CM6A-LR climate model. J. Adv. Model. Earth Syst. 12, e2019MS002010 (2020).

61. Gutjahr, O. et al. Max Planck Institute Earth System Model (MPI-ESM1.2) for the High-Resolution Model Intercomparison Project (HighResMIP). Geosci. Model Dev. 12, 3241-3281 (2019).

62. O'Neill, B. C. et al. The Scenario Model Intercomparison Project (ScenarioMIP) for CMIP6. Geosci. Model Dev. 9, 3461-3482 (2016).

63. World Development Indicators (The World Bank, 2020).

64. Fishery and Aquaculture Statistics: Global Production by Production Source, 1950-2018 (FAO, 2020).

65. Dyck, A. J. \& Sumaila, U. R. Economic impact of ocean fish populations in the global fishery. J. Bioecon. 12, 227-243 (2010).

66. Fishery and Aquaculture Statistics: Global Fisheries Commodities Production and Trade, 1976-2018 (FAO, 2020).

67. FAO Yearbook: Fishery and Aquaculture Statistics 2018 (FAO, 2020); https://doi.org/10.4060/cb1213t

68. Cisneros-Montemayor, A. M., Pauly, D., Weatherdon, L. V. \& Ota, Y. A global estimate of seafood consumption by coastal indigenous peoples. PLOS ONE 11, e0166681 (2016)

69. Kaufmann, D. \& Kraay, A. Worldwide Governance Indicators (The World Bank, 2018)

70. UN SDG Indicators (United Nations, 2020).

71. Demirgüç-Kunt, A., Klapper, L., Singer, D., Ansar, S. \& Hess, J. The Global Findex Database 2017: Measuring Financial Inclusion and the Fintech Revolution (The World Bank, 2018); https://doi.org/10.1596/978-1-4648 1259-0

72. Sea Around Us Concepts, Design and Data (Sea Around Us, 2020); seaaroundus.org

73. FAOSTAT Database (FAO, 2019)

74. Wendling, Z. A. et al. 2020 Environmental Performance Index (Yale Center for Environmental Law \& Policy, 2020); http://epi.yale.edu 
75. Cheung, W. W. L., Jones, M. C., Reygondeau, G. \& Frölicher, T. L. Opportunities for climate-risk reduction through effective fisheries management. Glob. Change Biol. 24, 5149-5163 (2018).

76. Jones, M. C. \& Cheung, W. W. L. Using fuzzy logic to determine the vulnerability of marine species to climate change. Glob. Change Biol. 24, e719-e731 (2018).

77. Tigchelaar, M. et al. Projected climate risk of aquatic food system benefits. Dryad https://doi.org/10.5061/dryad.70rxwdbz3 (2021).

78. World Economic Situation and Prospects 2020 - Statistical Annex (United Nations, 2020); https://www.un.org/development/desa/dpad/wp-content/ uploads/sites/45/WESP2020_Annex.pdf

\section{Acknowledgements}

This paper is part of the Blue Food Assessment (https://www.bluefood.earth/), a comprehensive examination of the role of aquatic foods in building healthy, sustainable and equitable food systems. The assessment was supported by the Builders Initiative, the MAVA Foundation, the Oak Foundation and the Walton Family Foundation, and it has benefited from the intellectual input of a wider group of scientists leading other components of the Blue Food Assessment work. Individual co-authors were supported by the CGIAR Research Program on Fish (E.Y.M. and M.J.P.); the CGIAR Research Program on Climate Change, Agriculture and Food Security (E.Y.M. and M.J.P.); the Swiss National Science Foundation (T.L.F., grant no. PP00P2_198897); the National Science Foundation (C.D.G., grant no. CNH 1826668); the John and Katie Hansen Family Foundation (C.D.G.); the Nippon Foundation Ocean Nexus Program (E.H.A.); Earthlab at the University of Washington (E.H.A.); the OceanCanada Partnership (U.R.S.); and the Social Science and Humanities Research Council of Canada (U.R.S.). We thank M. Aschwanden, A. Cisneros-Montemayor, S. Funge-Smith, J. Gee, D. Siegel and A. Thorpe for help accessing and analysing data.

\section{Author contributions}

M. Tigchelaar, W.W.L.C., E.Y.M. and M.J.P. co-led this study. M. Tigchelaar and W.W.L.C. conceived of the idea and designed the overall study together with E.Y.M., M.J.P. and E.R.S. M. Tigchelaar and H.J.P. reviewed the literature. M. Tigchelaar, W.W.L.C., M.A.O., T.L.F., J.A.G. and C.D.G. compiled the data. M. Tigchelaar and W.W.L.C. developed the model and analysed the data. M. Tigchelaar, W.W.L.C., E.Y.M., M.J.P., E.R.S., C.C.C.W., E.H.A. and A.B. conceived of the policy analysis and discussion. All authors reviewed the results and developed the main conclusions. M. Tigchelaar, W.W.L.C., E.Y.M. and M.J.P. wrote the manuscript draft, and all authors contributed to and approved the final manuscript.

\section{Competing interests}

The authors declare no competing interests.

\section{Additional information}

Extended data is available for this paper at https://doi.org/10.1038/s43016-021-00368-9.

Supplementary information The online version contains supplementary material available at https://doi.org/10.1038/s43016-021-00368-9.

Correspondence and requests for materials should be addressed to Michelle Tigchelaar Peer review information Nature Food thanks William Travis, Salvador Lluch-Cota and the other, anonymous, reviewer(s) for their contribution to the peer review of this work.

Reprints and permissions information is available at www.nature.com/reprints.

Publisher's note Springer Nature remains neutral with regard to jurisdictional claims in published maps and institutional affiliations.

(c) The Author(s), under exclusive licence to Springer Nature Limited 2021 


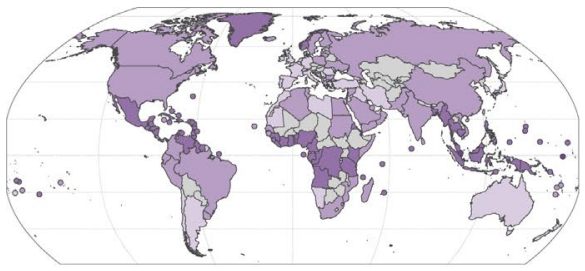

Hazard scores for scenario SSP5-8.5 in 2050
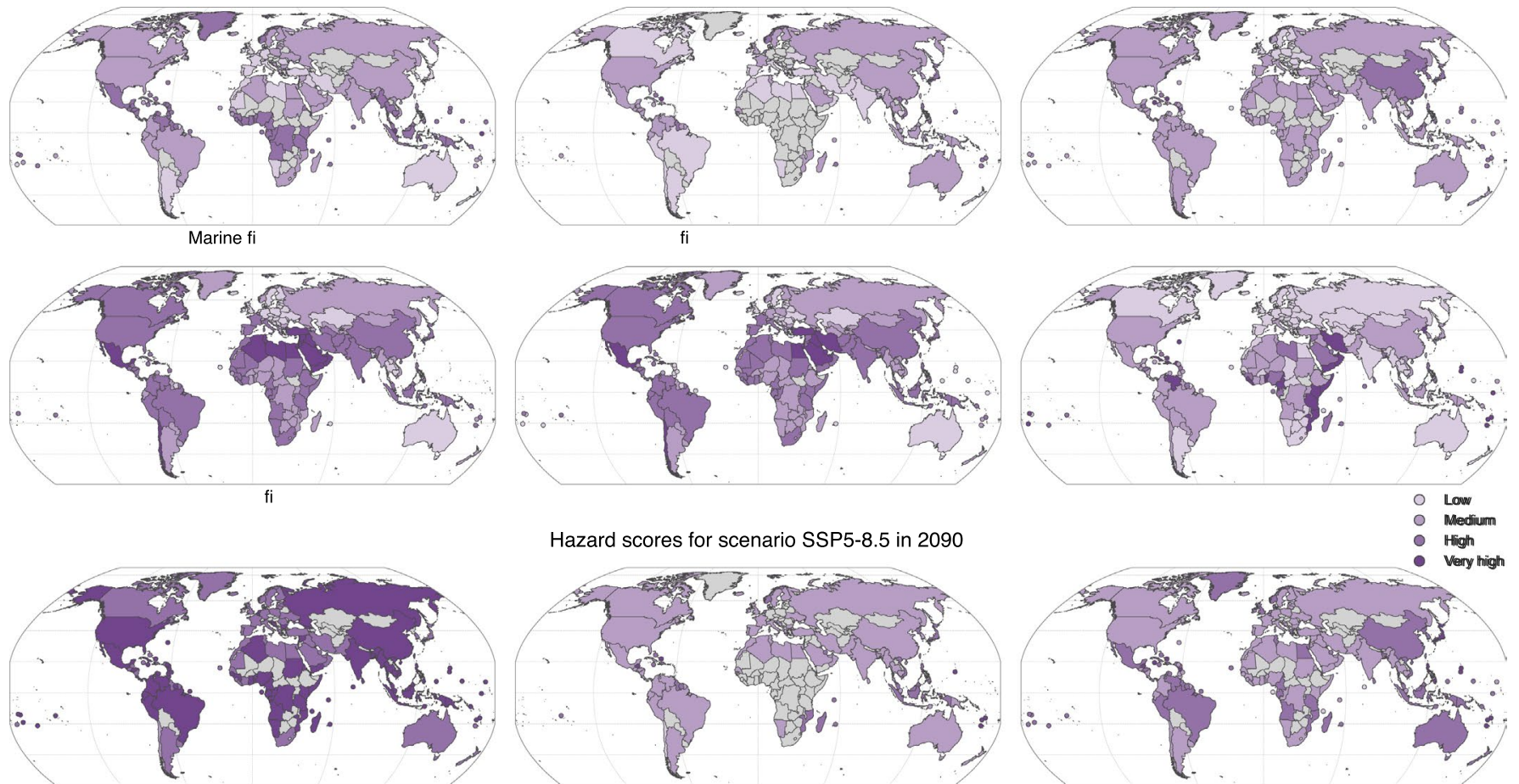

Hazard scores for scenario SSP5-8.5 in 2090

- Herny thighth

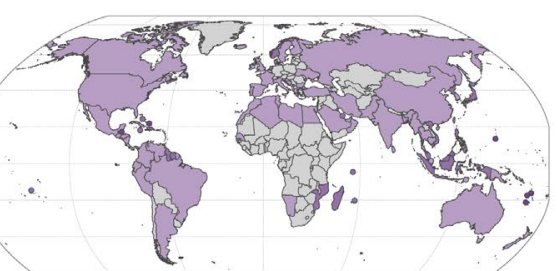

Marine aquaculture
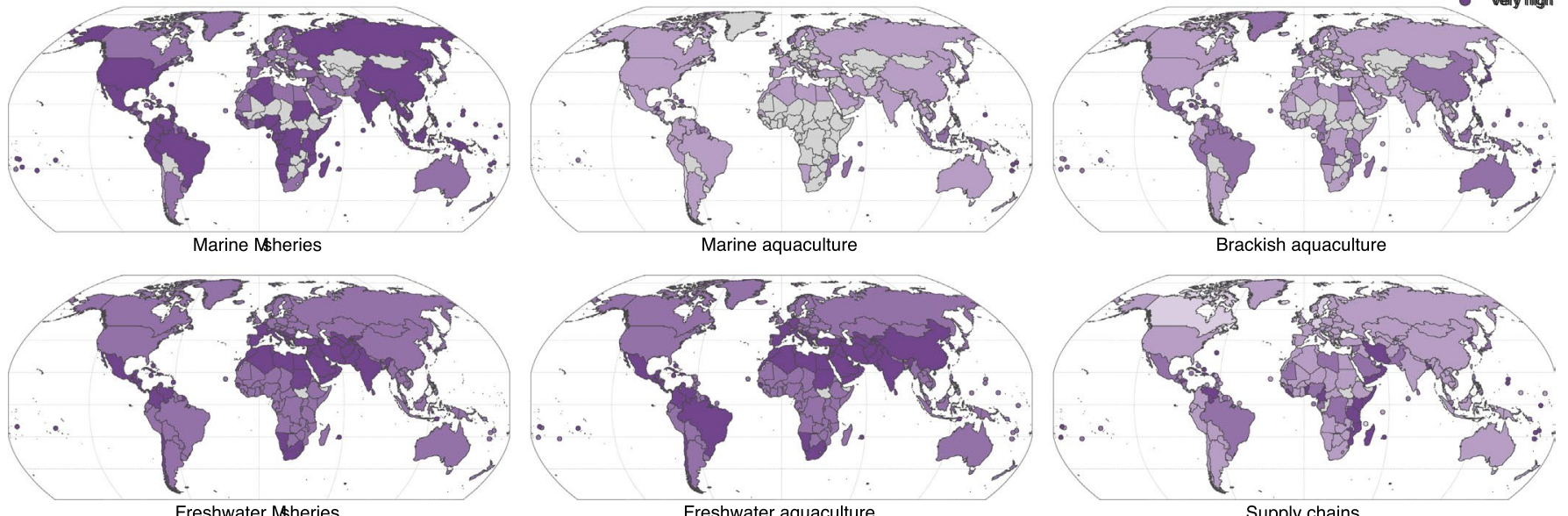

Freshwater Msheries

Freshwater aquaculture

Supply chains

Extended Data Fig. 1 | Projected climate hazard scores by food system component. Hazard scores for each of the aquatic food system components under a high-emissions scenario (SSP5-8.5) in 2041-2060 ('2050', top) and 2081-2100 ('2090', bottom). Gray means no data. Scores <25 are classified as 'low', 25-50 as 'medium', 50-75 as 'high', >75 as 'very high'. 

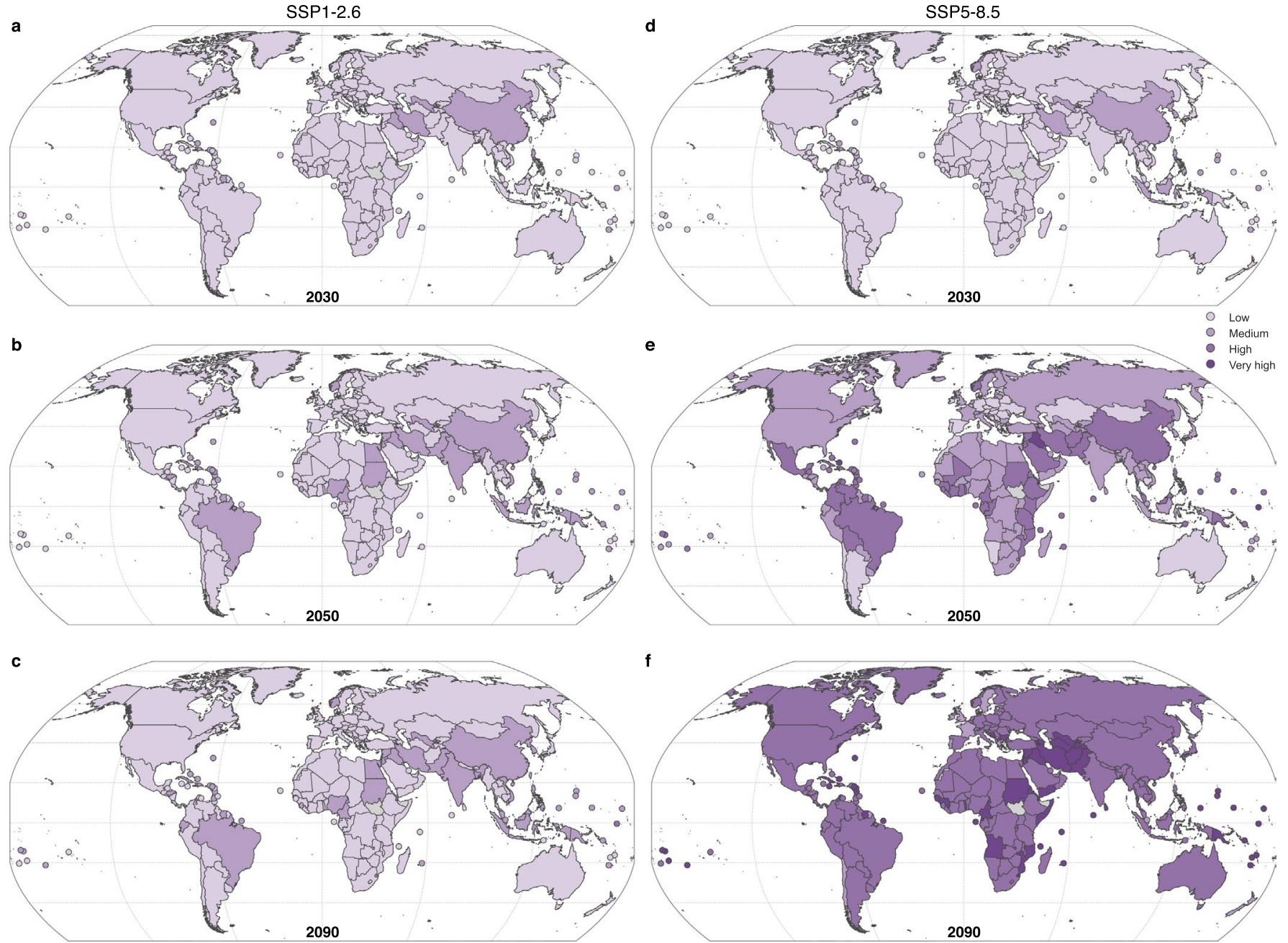

Extended Data Fig. 2 | Projected climate hazard scores in different scenarios and timeframes. Aggregate hazard score based on present-day production weights for a low-emissions scenario (SSP1-2.6, left) and a high-emissions scenario (SSP5-8.5, right) in 2021-2040 ('2030', top), 2041-2060 ('2050', middle) and 2081-2100 ('2090', bottom). a. SSP1-2.6 in 2030. b. SSP1-2.6 in 2050. c. SSP1-2.6 in 2090. d. SSP5-8.5 in 2030. e. SSP5-8.5 in 2050. f. SSP58.5 in 2090. Gray means no data. Scores <25 are classified as 'low', 25-50 as 'medium', 50-75 as 'high', > 75 as 'very high'. 
a
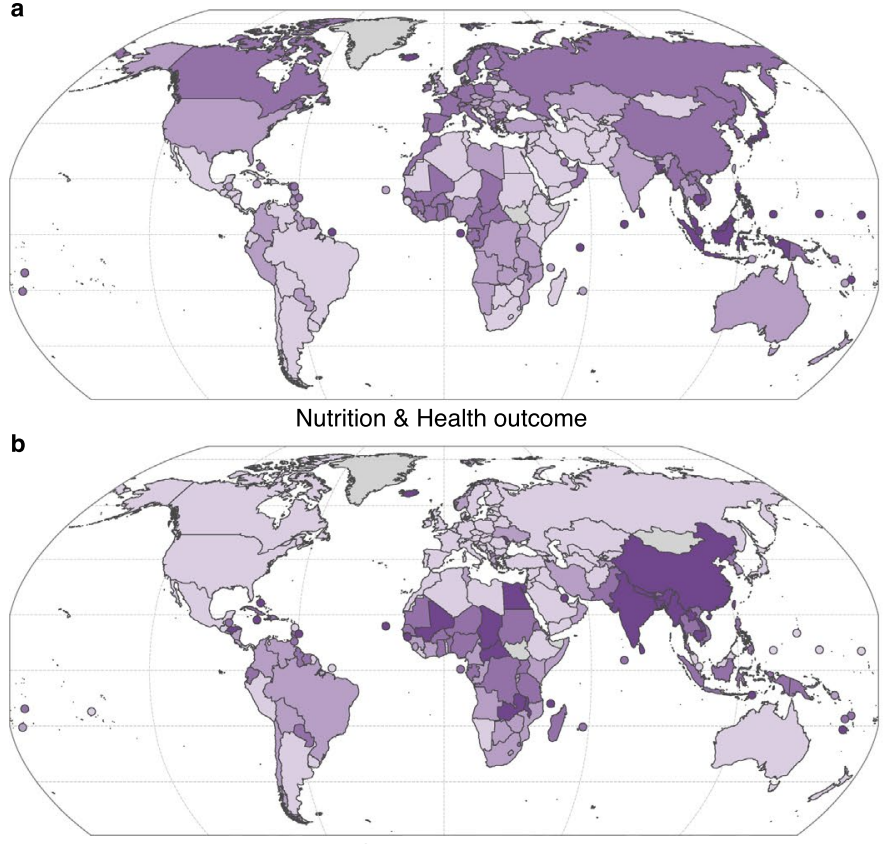

b

Social outcome c
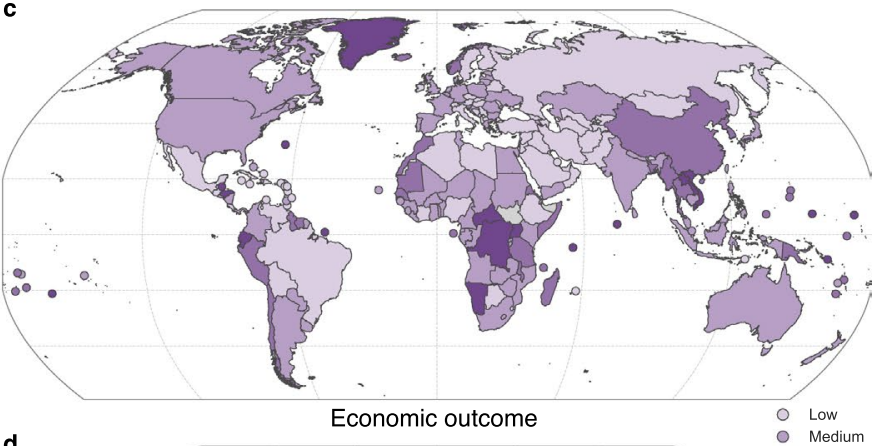

d

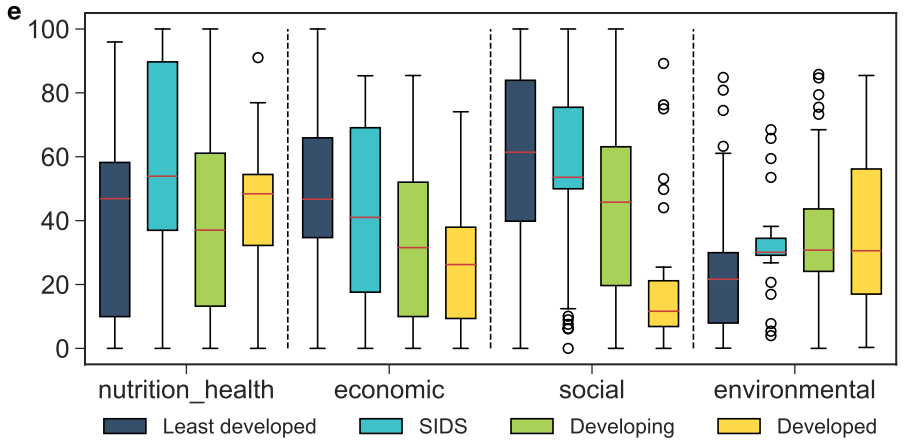

Extended Data Fig. 3 | Exposure scores for each of the food system outcomes. a. Scores for nutrition and health outcome; b. Scores for social outcome; c. Scores for economic outcome; d. Scores for environmental outcome; e. Exposure scores by development status. Gray means no data. Scores <25 are classified as 'low', 25-50 as 'medium', 50-75 as 'high', >75 as 'very high'. Box limits denote 25th and 75th percentiles; whiskers extend 1.5x the interquartile range from box edges; red line indicates median value and circles represent outliers. 
a
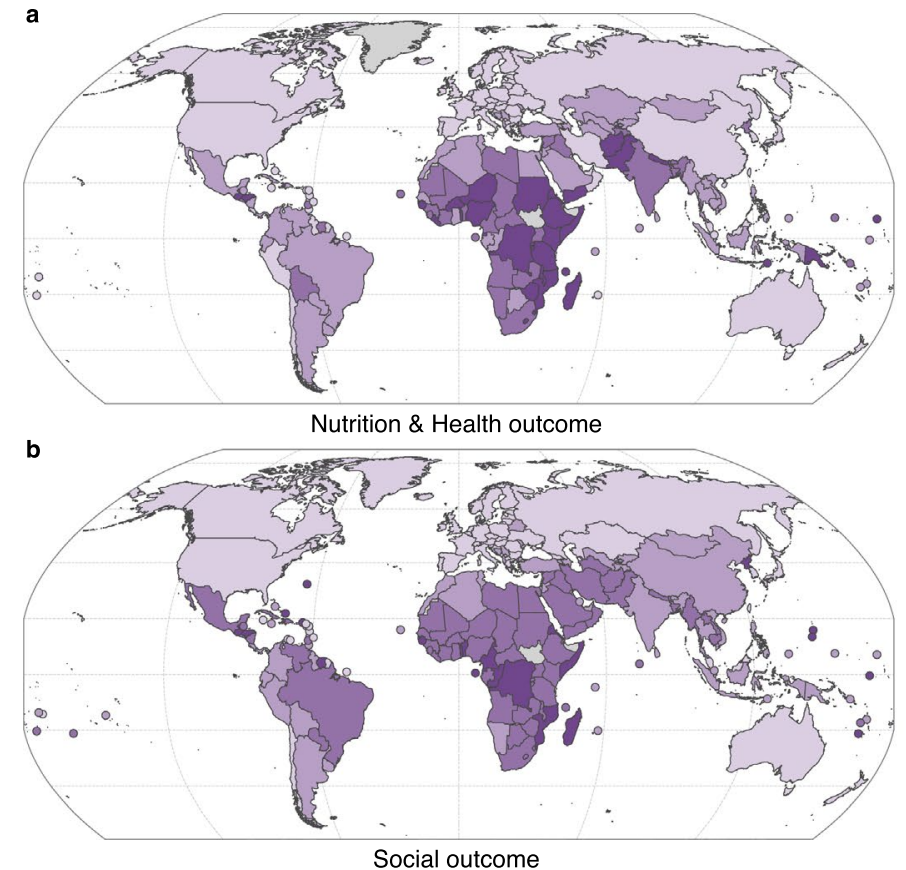

c
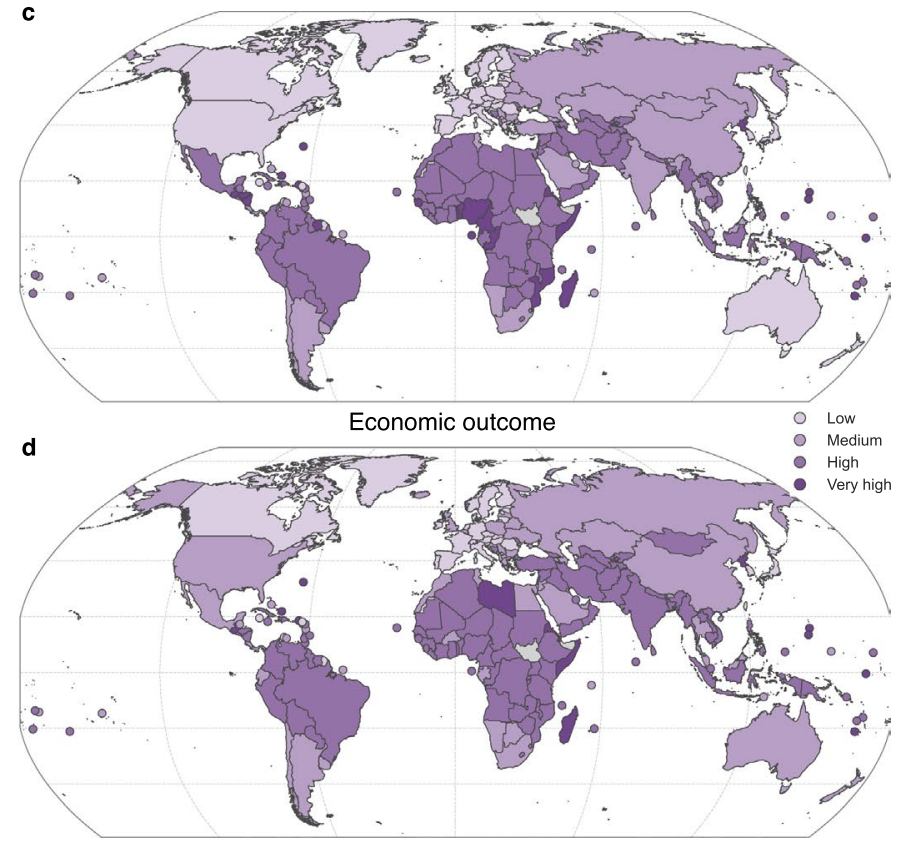

Environmental outcome

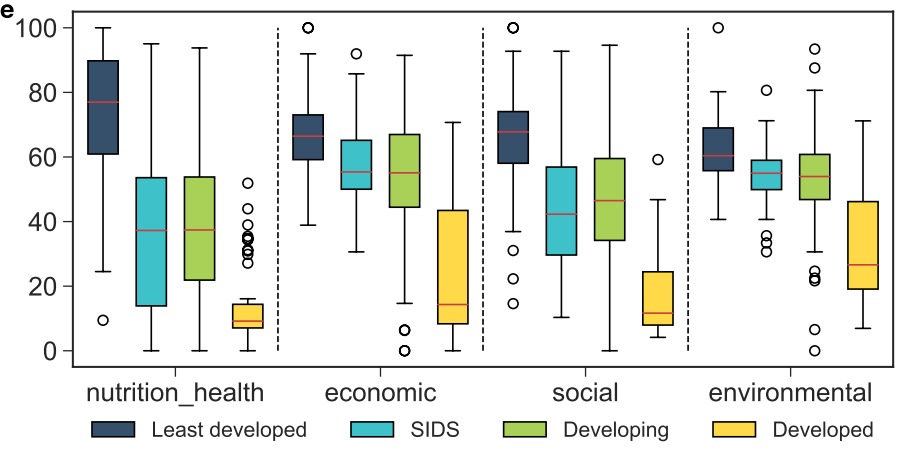

Extended Data Fig. 4 | Vulnerability scores for each of the food system outcomes. a. Scores for nutrition and health outcome; $\mathbf{b}$. Scores for social outcome; c. Scores for economic outcome; $\mathbf{d}$. Scores for environmental outcome; e. Vulnerability scores by development status. Gray means no data. Scores $<25$ are classified as 'low', 25-50 as 'medium', 50-75 as 'high', $>75$ as 'very high'. Box limits denote 25th and 75th percentiles; whiskers extend 1.5x the interquartile range from box edges; red line indicates median value and circles represent outliers. 

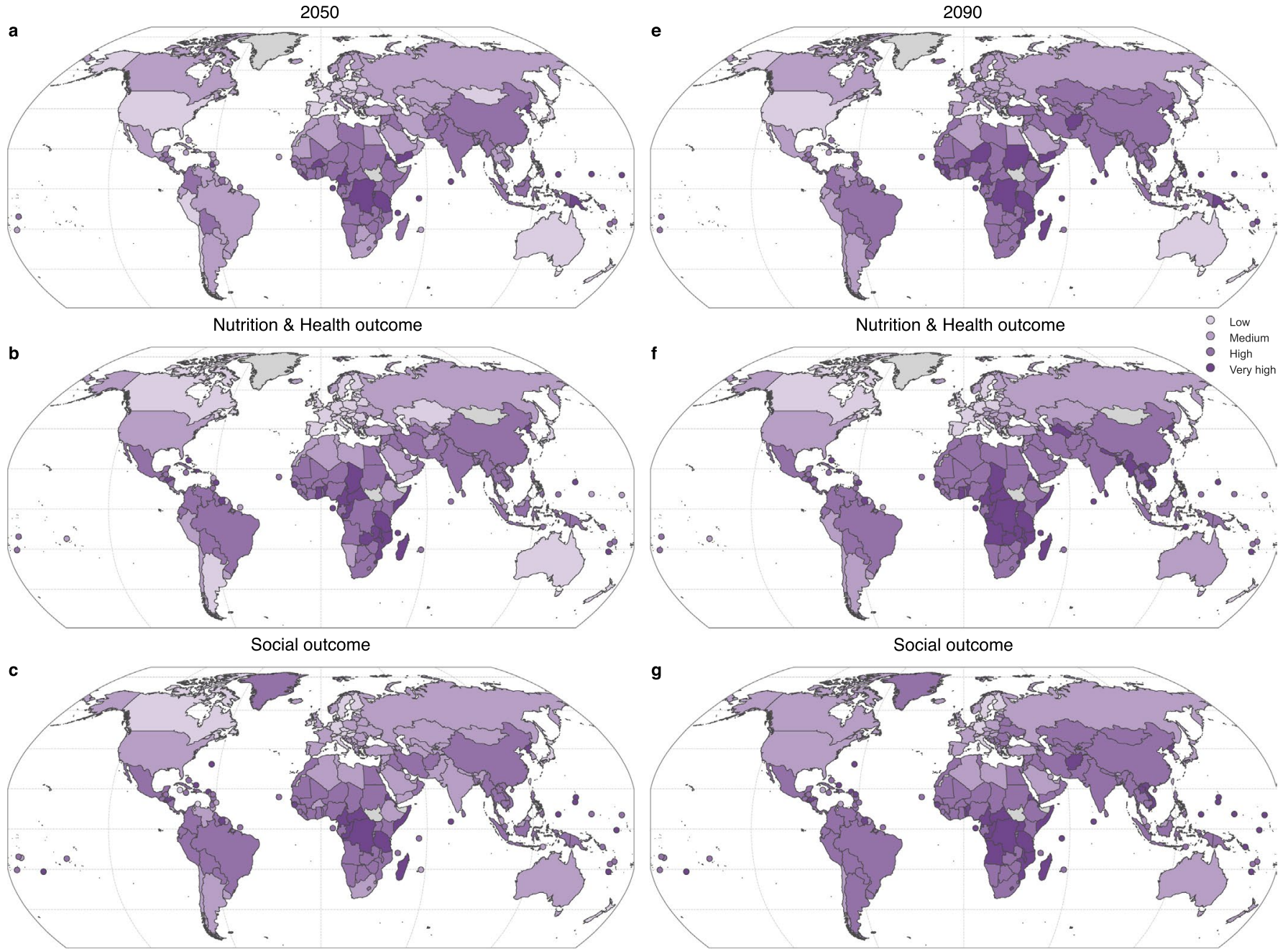

Economic outcome
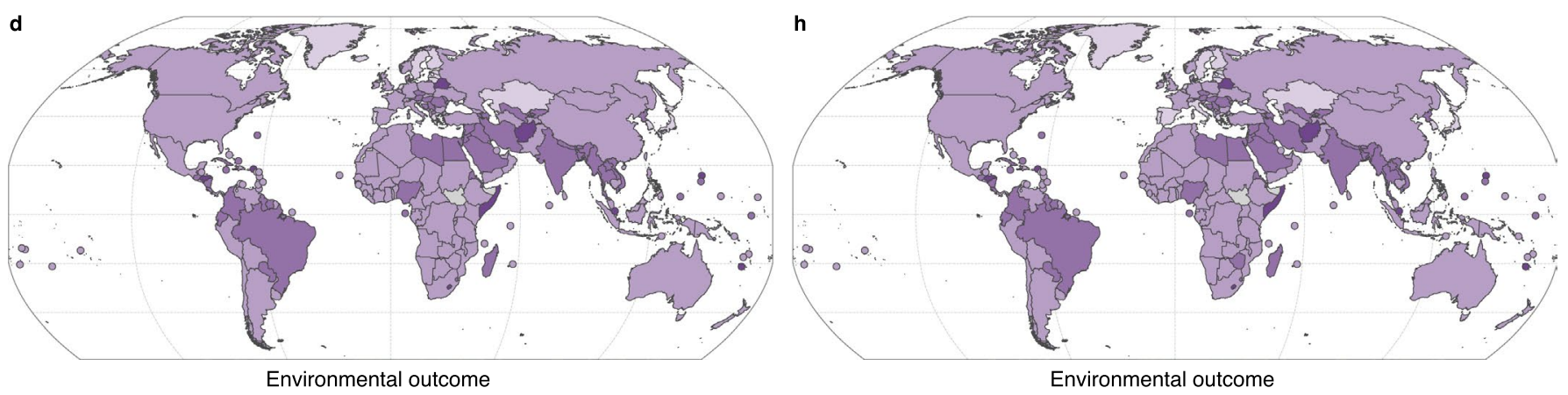

Extended Data Fig. 5 | Risk scores for each of the food system outcomes under high emissions. Projected climate risk for a high-emissions scenario (SSP5-8.5) in 2041-2060 ('2050', left) and 2081-2100 ('2090', right). a and e. Scores for nutrition and health outcome; b and f. Scores for social outcome; c and g. Scores for economic outcome; $\mathbf{d}$ and h. Scores for environmental outcome. Gray means no data. Scores $<25$ are classified as 'low', $25-50$ as 'medium', 50-75 as 'high', >75 as 'very high'. 


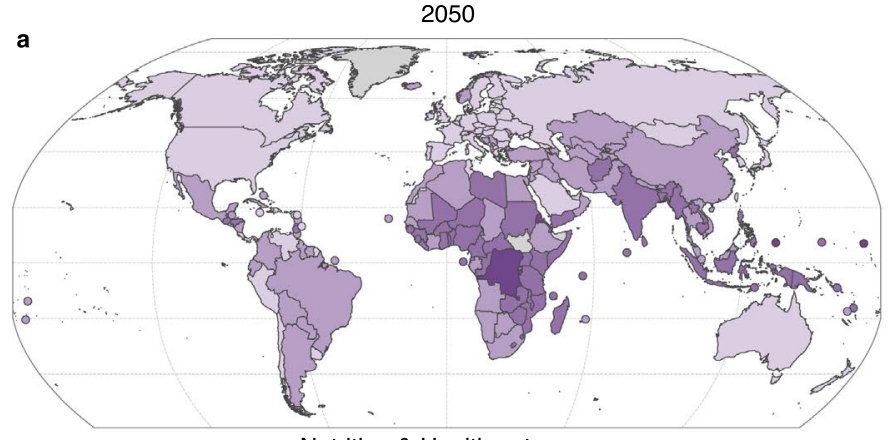

Nutrition \& Health outcome

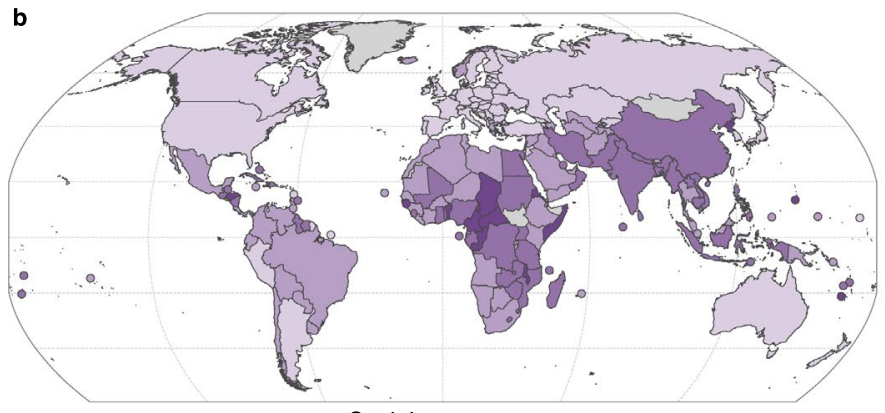

Social outcome

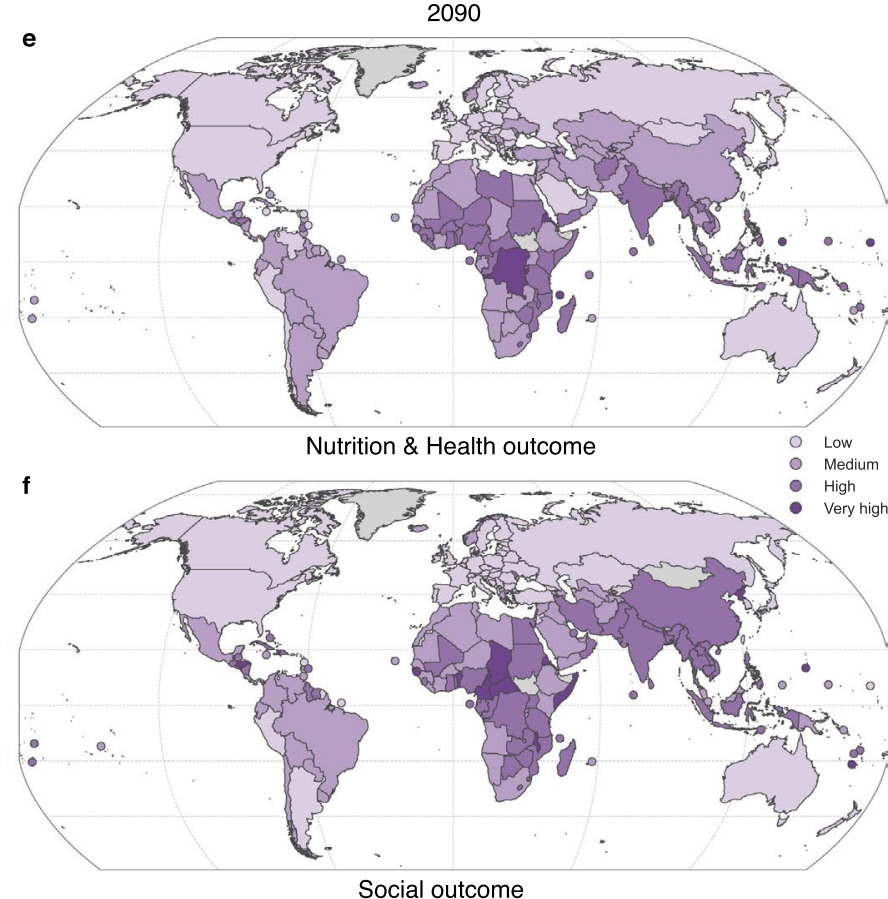

c
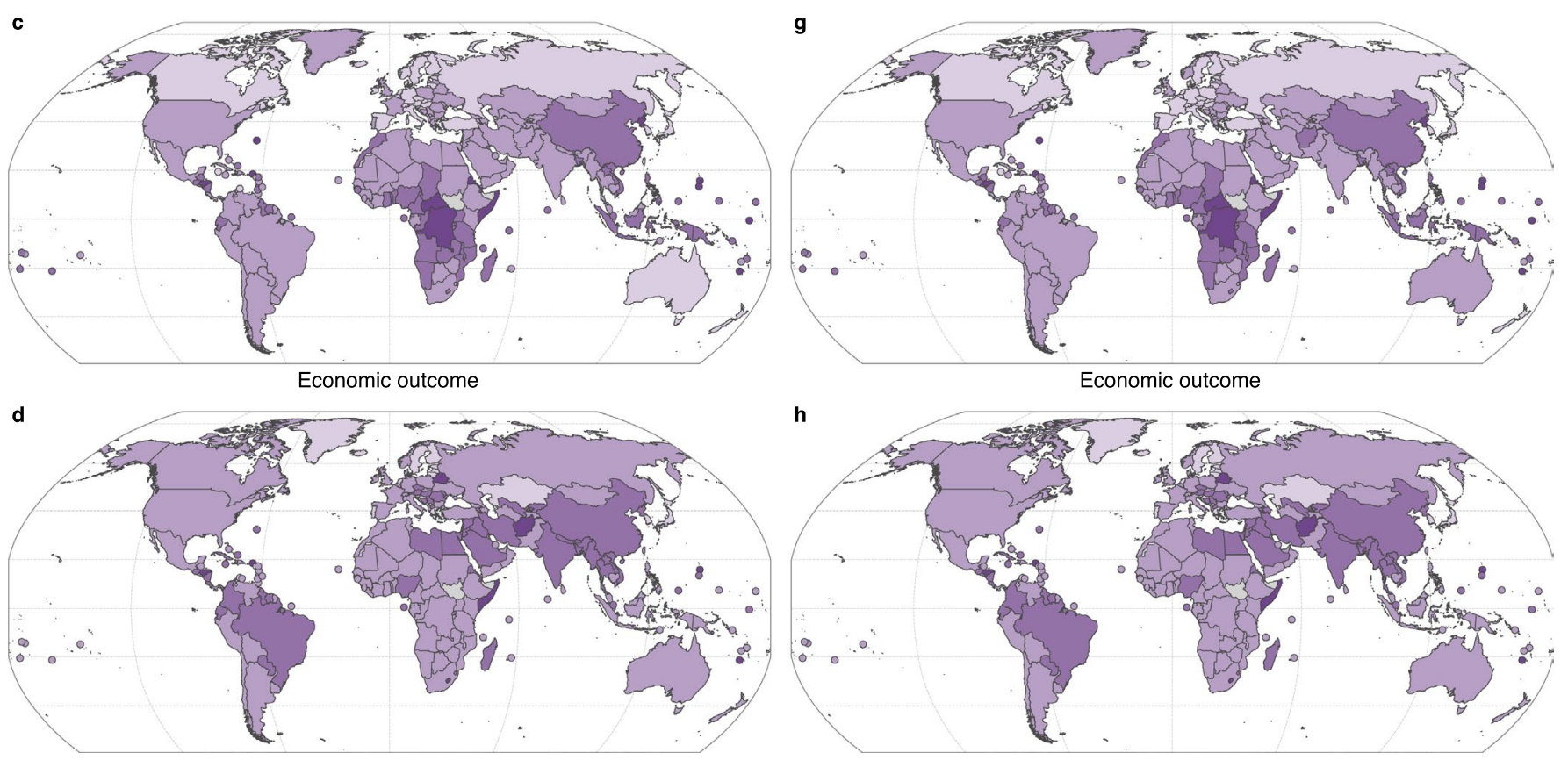

Environmental outcome

Environmental outcome

Extended Data Fig. 6 | Risk scores for each of the food system outcomes under low emissions. Projected climate risk for a low-emissions scenario (SSP12.6) in 2041-2060 ('2050', left) and 2081-2100 ('2090', right). a and e. Scores for nutrition and health outcome; b and f. Scores for social outcome; c and g. Scores for economic outcome; d and h. Scores for environmental outcome. Gray means no data. Scores <25 are classified as 'low', 25-50 as 'medium', $50-75$ as 'high', $>75$ as 'very high'. 
riskcount

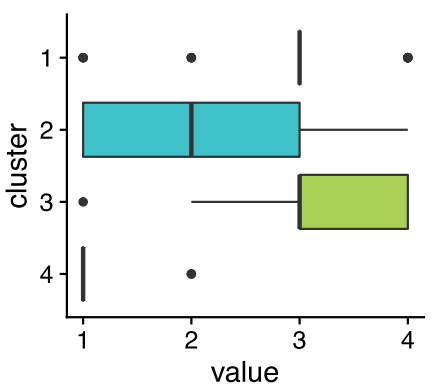

exp_nh

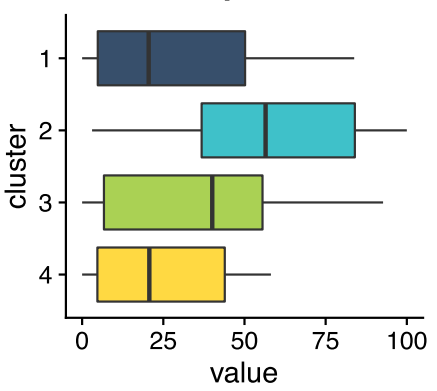

vul_nh

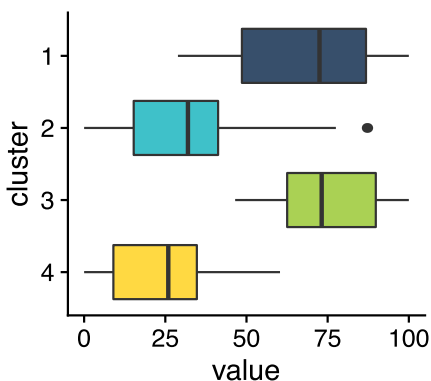

hazard

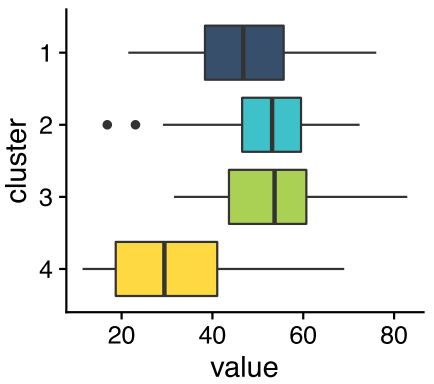

exp_ec

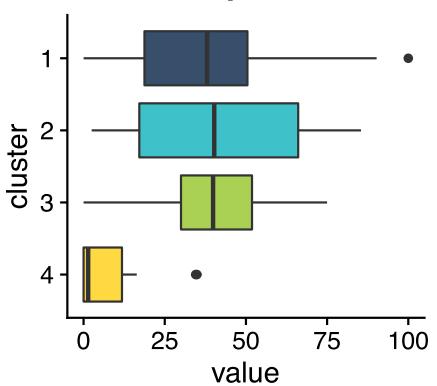

vul_ec

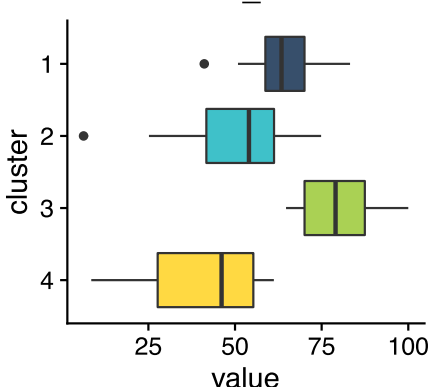

perc_marine

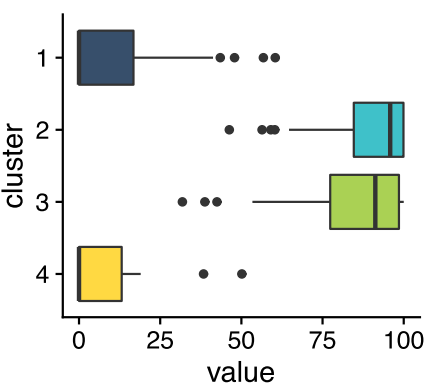

exp_so

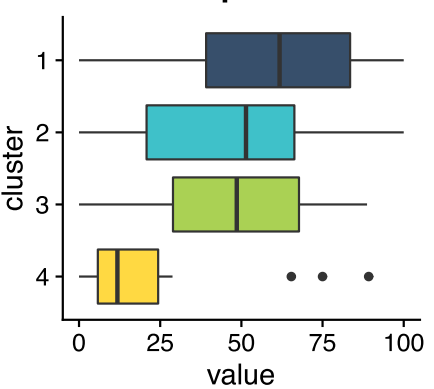

vul_so

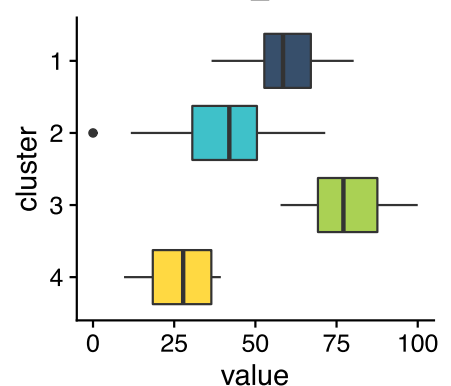

perc_aquaculture

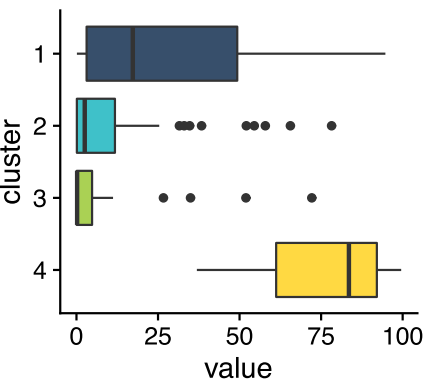

exp_en

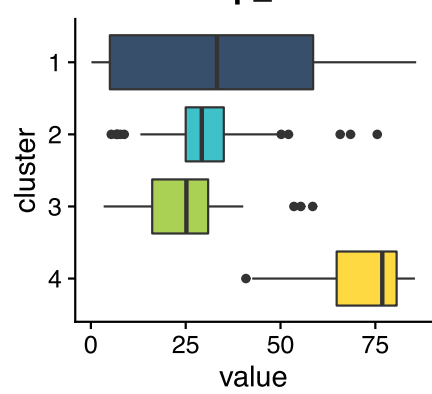

vul_en

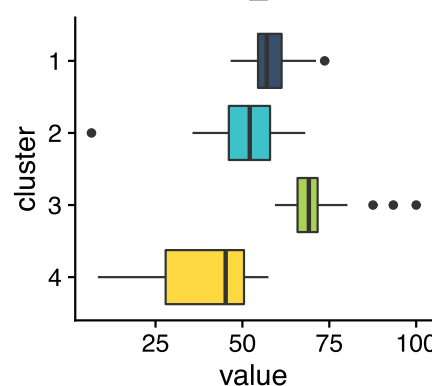

Extended Data Fig. 7 | Distribution of input variables for each climate risk cluster. The variables used in the cluster analysis are the number of outcomes for which climate risk in 2050 is 'high' or 'very high' under SSP5-8.5 ('riskcount'); the climate hazard score in 2050 under SSP5-8.5 ('hazard'); the percentage of production from marine environments ('perc_marine'); the percentage of production from aquaculture ('perc_aqua'); exposure scores for each of the food system outcomes (nutrition and health, economic, social, and environmental - 'exp_nh', 'exp_ec', 'exp_so' and 'exp_en'); and vulnerability scores for each of the food system outcomes ('vul_nh', 'vul_ec', 'vul_so' and 'vul_en'). We summarize the clusters as: Cluster $1=$ Compound risk -

freshwater \& deltaic system; Cluster 2 = High marine dependence; Cluster 3 = Compound risk - marine fisheries; Cluster $4=$ Environmental performance risk. Box limits denote 25th and 75th percentiles; whiskers extend 1.5x the interquartile range from box edges; black line indicates median value and circles represent outliers. 


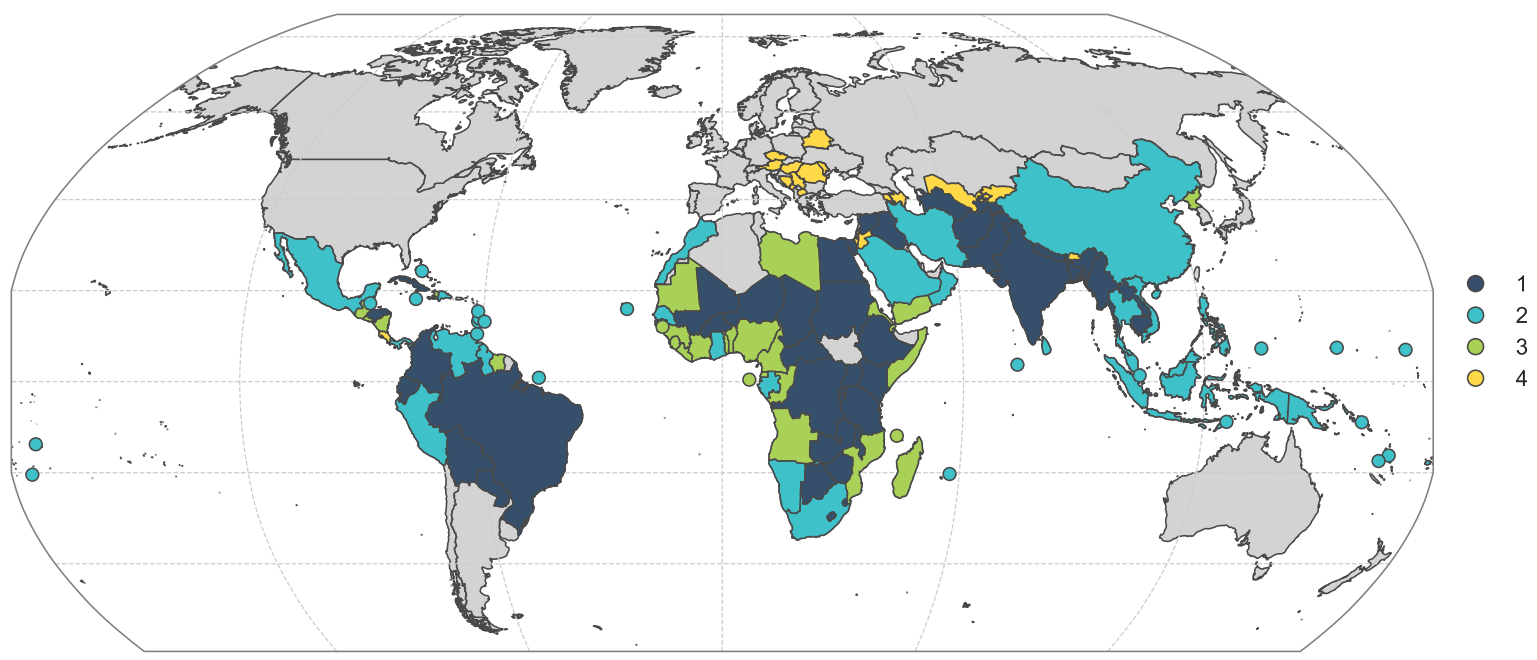

Extended Data Fig. 8 | Cluster assignment for each of the high risk countries. Cluster analysis was done only on countries that are projected to have 'high' or 'very high' risk in at least one of the food system outcomes and that had no missing data for any of the input variables. Cluster numbers correspond to the numbers in Extended Data Fig. 7 and the Supplemental Methods. Cluster $1=$ Compound risk - freshwater \& deltaic system; Cluster $2=$ High marine dependence; Cluster 3 = Compound risk - marine fisheries; Cluster 4 = Environmental performance risk. Grey shading represents no data. 